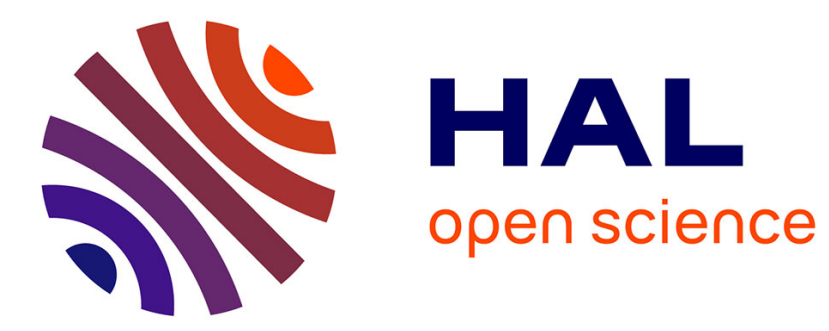

\title{
Scattering of a surface plasmon polariton by a surface defect
}

\author{
F. Pincemin, A. A. A Maradudin, A. D. Boardman, J.-J. Greffet
}

\section{To cite this version:}

F. Pincemin, A. A. A Maradudin, A. D. Boardman, J.-J. Greffet. Scattering of a surface plasmon polariton by a surface defect. Physical Review B: Condensed Matter and Materials Physics (19982015), 1994, 50 (20), pp.15261-15275. 10.1103/PhysRevB.50.15261 . hal-01617934

\section{HAL Id: hal-01617934 \\ https://hal.science/hal-01617934}

Submitted on 17 Oct 2017

HAL is a multi-disciplinary open access archive for the deposit and dissemination of scientific research documents, whether they are published or not. The documents may come from teaching and research institutions in France or abroad, or from public or private research centers.
L'archive ouverte pluridisciplinaire HAL, est destinée au dépôt et à la diffusion de documents scientifiques de niveau recherche, publiés ou non, émanant des établissements d'enseignement et de recherche français ou étrangers, des laboratoires publics ou privés. 


\title{
Scattering of a surface plasmon polariton by a surface defect
}

\author{
F. Pincemin* and A. A. Maradudin \\ Department of Physics and Institute for Surface and Interface Science, University of California, Irvine, California 92717
}

A. D. Boardman

Department of Physics, University of Salford, Salford M5 4WT, United Kingdom

J.-J. Greffet

Laboratoire d'Energétique Moléculaire et Macroscopique, Combustion, Ecole Centrale Paris, Châtenay-Malabry, 92295 Cedex, France

(Received 12 May 1994)

\begin{abstract}
By means of a Green-function, volume-integral-equation approach we study numerically the scattering of a surface plasmon polariton at a planar vacuum-metal interface by dielectric and metallic defects that are either embedded in the metal substrate or are situated in the vacuum region on the substrate. We calculate the transmission and reflection coefficients for the surface plasmon polariton, as well as the efficiency of its conversion into volume electromagnetic waves in the vacuum propagating away from the surface. We also compute the near field in the vicinity of the surface defect.
\end{abstract}

\section{INTRODUCTION}

The theoretical study of the scattering of surface waves by a surface defect had its origins in the work of Dean ${ }^{1}$ and Ursell, ${ }^{2,3}$ who showed that a surface wave incident normally upon a rigid circular cylinder submerged in an incompressible fluid of infinite depth passes over the cylinder with a change of phase but without a change of amplitude, and experiences no reflection, whatever the frequency of the incident wave, for any values of the radius and depth of the cylinder.

Subsequently, Levine ${ }^{4}$ extended this work to the case of oblique incidence, by the use of approximate methods in the limit of a small radius of the cylinder, and showed that in this case a partial reflection of the surface wave usually occurs.

Davis and Hood ${ }^{5}$ showed that if the cylinder has an arbitrary, noncircular, cross section, and if the depth of the fluid is possibly finite, a vanishing reflection of the incident surface wave occurs only for certain combinations of the frequency of the wave and geometry of the fluid system. They also showed that if the cross section of the cylinder is not circular, and the depth of the fluid is infinite, there will be a reflected wave in general.

In contrast with the preceding studies, in which a surface wave was scattered by a single cylinder submerged in a fluid, Davis and Leppington ${ }^{6}$ studied a surface wave traveling under the influence of gravity along the surface of a body of fluid and scattered by one or more totally immersed cylinders.

The results for the scattering of surface waves by cylinders of different cross sections, either completely or partially submerged in a fluid of infinite or finite depth, in particular the absence of reflection from a cylinder of circular cross section in a fluid of infinite depth, stimulated investigations of whether an analogous phenomenon ex- ists for surface electromagnetic waves. The first such study was carried out by Cullen. ${ }^{7} \mathrm{He}$ considered the case of a plane, TM ( $p$-polarized) surface electromagnetic wave, propagating over a corrugated metal surface, and incident on an infinitely long cylinder of elliptic cross section, whose axis is parallel to the magnetic field, and whose cross-section dimensions are small compared to the wavelength of the surface wave. By the use of an impedance boundary condition at the metal surface, he showed that if the elliptic cylinder is perfectly conducting, and if the ratio of the semimajor and semiminor axes of the cross section of the cylinder is suitably chosen, there exists one specific frequency of the surface wave at which the reflected wave vanishes.

This work was followed by that of Davis and Leppington, ${ }^{8}$ who also considered the case of a $p$-polarized, plane surface electromagnetic wave, that propagates across a corrugated metal surface, and is scattered by a cylinder of circular or elliptic cross section. The cylinder was taken to be either a perfect conductor or a dielectric with zero or finite conductivity. Its width was assumed to be small compared to the other length scales in the problem. An impedance boundary condition was assumed on the metal surface traversed by the surface wave. It twas found that, in general, the wave is both partially reflected and partially converted into radiative volume electromagnetic waves, in addition to being transmitted as a surface wave, and calculated the reflection and transmission coefficients of the surface wave for various combinations of the geometrical and material properties of the scattering system.

Whereas in the studies cited up to this point a surface electromagnetic wave was scattered by a subsurface, infinitely long cylinder, in a subsequent paper Davis and Leppington ${ }^{9}$ studied the scattering by a perfectly conducting sphere of a surface electromagnetic wave that 
travels along a corrugated surface between parallel, perfectly conducting walls. The reflection and transmission coefficients of the surface wave were obtained in this work. In a more recent follow-up to this study, Davis ${ }^{10}$ investigated a surface electromagnetic wave traveling between perfectly conducting walls and incident on a small, axisymmetric, dielectric spheroid. The reflection and transmission coefficients of the surface wave were calculated by a generalization of the method of Ref. 9, and the results were used in an investigation of the possibility of zero reflection of the incident wave.

In recent work, prompted in large measure by a desire to establish a spectroscopy of solid surfaces based on surface electromagnetic waves rather than on volume electromagnetic waves, due to the greater sensitivity of the former to surface conditions, attention has been directed to the scattering of $p$-polarized surface electromagnetic waves by dielectric films deposited on metallic substrates. Agranovich, Kravtsov, and Leskova ${ }^{11,12}$ studied the scattering of a surface electromagnetic wave at a vacuum-metal interface that is incident normally on a thin dielectric film that covers one half the surface of the metal. The metal substrate and the semi-infinite dielectric film were represented by a coordinate-dependent impedance boundary condition satisfied on the surface of the metal, which is exact to first order in $d / \lambda$, where $d$ is the thickness of the film and $\lambda$ is the wavelength of the surface electromagnetic wave. The frequency-dependent dielectric constant of the dielectric film was assumed to have a pole at a frequency $\omega_{0}$ of an electric dipole-active excitation (vibration) that falls in the frequency range in which surface electromagnetic waves at the metalvacuum interface exist. In this case, the portion of the surface covered by the dielectric film supports two surface electromagnetic waves. ${ }^{13}$ A portion of the energy of the incident surface wave is converted into these two surface waves. A portion is converted into a reflected surface wave on the metal-vacuum interface, and a portion is converted into volume waves in the vacuum above the structure. For frequencies of the incident surface wave near $\omega_{0}$ its conversion into volume waves was found to be enhanced, which effect can be used to obtain information about the vibrational spectrum of the film. Analogous calculations in the case that the dielectric film covers only a portion of the metal surface of length $L$ in the direction of propagation of the surface electromagnetic wave were carried out by Leskova and Gapotchenko. ${ }^{14}$ In this case, the transmission coefficient of the surface polariton displays a complex, oscillatory dependence on the length of the film $L$ caused by the interference of the two surface polaritons in the region of the film and by their multiple reflection from the edges of the film. However, the results presented by Leskova and Gapotchenko are valid only in the limit $k L \gg 1$, where $k$ is the wave number of the surface plasmon polariton.

The same calculations were carried out by means of a modal analysis by Stegeman et al. ${ }^{15}$ and by Shen et al. ${ }^{16}$ In this work, the metal surface covered by a semiinfinite $^{15}$ or a finite ${ }^{16}$ dielectric film was embedded in a waveguide bounded by perfectly conducting planes parallel to the surface of the metal, and far enough away from it that the electromagnetic field of the surface wave was sensibly unaffected by their presence. The spectrum of the electromagnetic modes in each of the segments of the waveguide corresponding to the uncoated and coated portions of the metal surface is made discrete by the introduction of the perfectly conducting planes, and the fields in each of the segments were expanded in these modes. The coefficients in these expansions were obtained by satisfying the electromagnetic boundary conditions at the vertical interfaces between the segments. The transmission and reflection coefficients for the surface electromagnetic waves are obtained quite accurately by this procedure, but the angular distribution of the intensity of the volume waves in the vacuum region generated by the interaction of the incident surface wave with the dielectric film is less well reproduced.

In a recent paper, ${ }^{17}$ the excitation of surface plasmons by a submerged cylinderical defect illuminated by a plane wave has been studied by a numerical approach based on the solution of a volume integral equation. The interference between the surface polaritons scattered by two different defects has been investigated, and it has been shown that surface polaritons can be almost localized between the defects for suitable values of the angle of incidence and of the distance between the defects. This study has been extended ${ }^{18}$ to the case of a large number of defects randomly distributed along the interface. For that system, the surface polaritons are multiply scattered. A backscattering enhancement peak is observed, which is the signature of the weak localization of surface polaritons.

As the preceding survey shows, the problem of the scattering of a surface electromagnetic wave by a surface defect has a long history. Nevertheless, it is still of current interest, and there are several motivations that prompt us to investigate this problem further in this paper.

The first motivation for this work is the desire to develop a method for computing without approximation the scattering of a surface electromagnetic wave-a surface plasmon polariton-at a planar vacuum-metal interface that is incident normally on an infinitely long, dielectric or metallic, cylindrical defect of arbitrary cross section. By a surface defect we will mean throughout this paper a defect that lies near the interface, either completely embedded in one or the other of the media in contact along it, or that intersects the interface.

Second, we are interested in pursuing a systematic analysis of the scattering of a surface plasmon polariton incident normally on a surface defect of rectangular cross section, whose length parallel to the direction of propagation of the waves is much larger than its thickness in the direction normal to the surface. In the case that the surface defect is a dielectric film deposited on a metallic substrate we seek results that are not restricted to films whose thickness is small in comparison to the wavelength of the surface polariton, and which describe accurately the angular dependence of the intensity of the volume waves in the vacuum generated by the interaction of the surface wave with the film. Such results can aid in the interpretation of results of experimental investigations of 
such scattering phenomena. ${ }^{19-21}$ At the same time, the exact formulation of surface polariton scattering problems developed here also enables us to study the scattering from a surface defect whose length in the direction of propagation, even if much larger than its thickness, is of the order of the vacuum wavelength of the surface polariton, in contrast with the work of Leskova and Gapotchenko. ${ }^{14}$ Such results may be of interest in the study of optoelectronic devices and in integrated optics, since the kinds of material and geometrical discontinuities represented by such defects will occur in any integrated optics circuits impressed on surfaces that employ surface polaritons.

The last main motivation for this work lies in the recent development of near field optics, which has stimulated the development of theoretical methods for studying the scattering of illuminating beams by given features of the surface such as dielectric contrast, microroughness, or the presence of a tip. ${ }^{22-24}$ As a consequence, we have also calculated in this paper the scattered electromagnetic field in the near field region in the vacuum, which would be detected by a nonperturbative probe.

In Sec. II we present the theory of the volumeintegral-equation method used in this paper. In Sec. III we present the results of our calculations for the scattering of a surface plasmon polariton by a long, rectangular surface defect. We look first at the case of a dielectric film of finite length deposited on a metallic substrate (Sec. III A), and then consider metallic particles (Sec. III B) and dielectric particles (Sec. III C) embedded in a metal. A discussion of the results obtained, and the conclusions reached from them, is presented in Sec. IV.

\section{THEORY}

In this section, we outline the derivation of the numerical approach to the computation of the scattered electromagnetic field when a surface electromagnetic wave is incident normally on a particle in the vicinity of the surface of a semi-infinite medium. The volume-integral approach on which this technique is based has already been described for the case that a $p$-polarized plane wave is incident from the vacuum on such a system. ${ }^{17}$ In the present article, the incident field is a surface electromagnetic wave, viz. a surface plasmon polariton. Nevertheless, the scattering theory in this case is very similar to that of Ref. 17. Consequently, we will only outline the general numerical technique used here, to make this paper self-contained, but will describe the treatment of the far field and near field and the way to compute the reflection and transmission coefficients of the surface plasmon polaritons in some detail.

\section{A. Geometry of the problem}

The unperturbed physical system underlying the work described in this paper consists of a linear dielectric medium characterized by an isotropic, dielectric constant $\epsilon_{1}=n_{1}^{2}$ in the region $z>0$, and a linear dielectric medium characterized by an isotropic, dielectric constant $\epsilon_{3}=n_{3}^{2}$ in the region $z<0$. The regions $z>0$ and $z<0$ will be denoted by $\Omega_{1}$ and $\Omega_{3}$, respectively. In what follows, properties of this reference system will be labeled by the subscript $f$. In particular, we denote by $\epsilon_{f}(z)$ its dielectric constant, which is defined by

$$
\epsilon_{f}(z)= \begin{cases}\epsilon_{1} & \text { for } z>0 \\ \epsilon_{3} & \text { for } z<0\end{cases}
$$

We assume that the interface $z=0$ separating these two media supports a p-polarized surface electromagnetic wave.

We will be concerned with the following two situations: (1) the region $\Omega_{1}$ is a lossless metal while the region $\Omega_{3}$ is a lossless dielectric and (2) the region $\Omega_{1}$ is a lossless dielectric while the region $\Omega_{3}$ is a lossless metal. The dielectric constants $\epsilon_{1}$ and $\epsilon_{3}$ are, therefore, both real in each of these two situations, and the surface electromagnetic wave supported by the surface $z=0$ is called a surface plasmon polariton. The conditions ensuring the existence of this surface wave are ${ }^{25}$

$$
\begin{aligned}
& \epsilon_{1} \epsilon_{3}<0, \\
& \epsilon_{1}+\epsilon_{3}<0 .
\end{aligned}
$$

We now introduce into this system a cylindrical particle of finite extent in both the $x$ and $z$ directions, whose generators are parallel to the $y$ axis. The resulting system is, therefore, invariant along the $y$ axis $(\partial / \partial y \equiv 0)$. The region of the $x z$ plane intercepted by the particle will be denoted by $\Omega_{2}$. The particle is characterized by an isotropic dielectric constant $\epsilon_{2}=n_{2}^{2}$, which is also assumed to be real. In what follows, we will consider particles present only in the semi-infinite region $z>0\left(\Omega_{1}\right)$. The geometry of the resulting structure, and the notation adopted, are shown in Fig. 1. The dielectric constant of the entire system will be denoted by $\epsilon(x, z)$.

The region $\Omega_{2}$ in which the dielectric constant $\epsilon(x, z)$ is different from $\epsilon_{f}(z)$ breaks the infinitesimal translational invariance of the system depicted in Fig. 1 along directions in the plane $z=0$, and scatters a surface plasmon polariton incident on it. We now turn to the study of this scattering.

\section{B. Determination of the field in the source region}

In this paper, all components of the electromagnetic field will be assumed to have a time dependence $\exp (-i \omega t)$ and we will write a typical component in the form

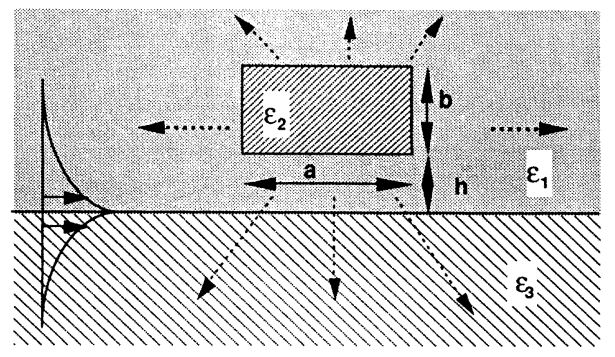

FIG. 1. Geometry of the system studied in this paper. 


$$
A_{i}(x, z ; t)=A_{i}(x, z \mid \omega) \exp (-i \omega t) .
$$

Let $\mathbf{E}_{f}(x, z \mid \omega)$ be the incident electric field, viz. a surface plasmon polariton, while $\mathbf{E}(x, z \mid \omega)$ is the total electric field. The latter satisfies the vector Helmholtz equation

$$
\nabla \times \nabla \times \mathbf{E}-\epsilon(x, z) k_{0}^{2} \mathbf{E}=0,
$$

where $k_{0}=\omega / c$. The incident field satisfies a similar equation

$$
\nabla \times \nabla \times \mathbf{E}_{f}-\epsilon_{f}(z) k_{0}^{2} \mathbf{E}_{f}=0 .
$$

By subtracting Eq. (2.5) from Eq. (2.4) we find that the scattered field $\mathbf{E}_{s}(x, z \mid \omega)=\mathbf{E}(x, z \mid \omega)-\mathbf{E}_{f}(x, z \mid \omega)$ satisfies the equation

$\nabla \times \nabla \times \mathbf{E}_{s}-\epsilon_{f}(z) k_{0}^{2} \mathbf{E}_{s}=\left[\epsilon(x, z)-\epsilon_{f}(z)\right] k_{0}^{2} \mathbf{E}$.

To solve this equation we introduce the Green dyadic $\overleftrightarrow{G}\left(x-x^{\prime} ; z, z^{\prime}\right)$, which is the solution of the equation

$$
\begin{array}{r}
\nabla \times \nabla \times \overleftrightarrow{G}\left(x-x^{\prime} ; z, z^{\prime}\right)-\epsilon_{f}(z) k_{0}^{2} \overleftrightarrow{G}\left(x-x^{\prime} ; z, z^{\prime}\right) \\
=\overleftrightarrow{I} \delta\left(x-x^{\prime}\right) \delta\left(z-z^{\prime}\right),
\end{array}
$$

where $\overleftrightarrow{I}$ is the unit dyad, subject to the radiation condition at infinity. By using Eqs. (2.6) and (2.7) we obtain the following integral equation for $\mathbf{E}(x, z \mid \omega)$ :

$$
\begin{aligned}
\mathbf{E}(x, z \mid \omega)= & \mathbf{E}_{f}(x, z \mid \omega) \\
& +k_{0}^{2} \int_{\Omega_{2}} d x^{\prime} d z^{\prime} \overleftrightarrow{G}\left(x-x^{\prime} ; z, z^{\prime}\right) \\
& \quad \times\left[\epsilon\left(x^{\prime}, z^{\prime}\right)-\epsilon_{f}\left(z^{\prime}\right)\right] \mathbf{E}\left(x^{\prime}, z^{\prime} \mid \omega\right) .
\end{aligned}
$$

The integration is carried out over the region in which $\epsilon(x, z)$ differs from $\epsilon_{f}(z)$, which is the region $\Omega_{2}$ occupied by the particle.

The incident field $\mathbf{E}_{f}(x, z \mid \omega)$ is that of a surface plasmon polariton, and is given $b y^{26}$

$$
\mathbf{E}_{f}(x, z \mid \omega)=\left[E_{f x}(x, z \mid \omega), 0, E_{f z}(x, z \mid \omega)\right],
$$

where, for $z>0$,

$$
\begin{aligned}
& E_{f x}(x, z \mid \omega)=E_{\mathrm{sp}}^{(0)} e^{i k_{\mathrm{sp}} x-\beta_{1} z} \\
& E_{f z}(x, z \mid \omega)=i \frac{k_{\mathrm{sp}}}{\beta_{1}} E_{\mathrm{sp}}^{(0)} e^{i k_{\mathrm{sp}} x-\beta_{1} z},
\end{aligned}
$$

while for $z<0$

$$
\begin{aligned}
& E_{f x}(x, z \mid \omega)=E_{\mathrm{sp}}^{(0)} e^{i k_{\mathrm{sp}} x+\beta_{3} z}, \\
& E_{f z}(x, z \mid \omega)=-i \frac{k_{\mathrm{sp}}}{\beta_{3}} E_{\mathrm{sp}}^{(0)} e^{i k_{\mathrm{sp}} x+\beta_{3} z} .
\end{aligned}
$$

In Eqs. (2.10)-(2.11), $E_{\mathrm{sp}}^{(0)}$ is a real amplitude and $k_{\mathrm{sp}}(\omega)$, $\beta_{1}(\omega)$, and $\beta_{3}(\omega)$ are given by

$$
\begin{aligned}
& k_{\mathrm{sp}}(\omega)=\frac{\omega}{c}\left(\frac{\epsilon_{1} \epsilon_{3}}{\epsilon_{1}+\epsilon_{3}}\right)^{1 / 2}, \\
& \beta_{1}(\omega)=\left[k_{\mathrm{sp}}^{2}-\epsilon_{1} \frac{\omega^{2}}{c^{2}}\right)^{1 / 2}=\frac{\omega}{c}\left(\frac{-\epsilon_{1}^{2}}{\epsilon_{1}+\epsilon_{3}}\right)^{1 / 2}, \\
& \beta_{3}(\omega)=\left(k_{\mathrm{sp}}^{2}-\epsilon_{3} \frac{\omega^{2}}{c^{2}}\right)^{1 / 2}=\frac{\omega}{c}\left(\frac{-\epsilon_{3}^{2}}{\epsilon_{1}+\epsilon_{3}}\right)^{1 / 2} .
\end{aligned}
$$

We will also need the result that for $z>0$

$$
H_{f y}(x, z \mid \omega)=\frac{\omega \epsilon_{1}}{i c \beta_{1}} E_{\mathrm{sp}}^{(0)} e^{i k_{\mathrm{sp}} x-\beta_{1} z},
$$

while for $z<0$

$$
H_{f y}(x, z \mid \omega)=-\frac{\omega \epsilon_{3}}{i c \beta_{3}} E_{\mathrm{sp}}^{(0)} e^{i k_{\mathrm{sp}} x+\beta_{3} z}
$$

We solve the integral equation $(2.8)$ by the method of moments. ${ }^{27}$ By assuming that the particle can be mapped into a set of rectangular cells of dimensions $\Delta x$ and $\Delta z$ parallel to the $x$ and $z$ axes, respectively, and that the electric field can be considered constant throughout each cell, we obtain from Eq. (2.8) the linear system

$$
\begin{aligned}
& E_{x}^{i}=E_{f x}^{i}+M_{x x}^{i j} E_{x}^{j}+M_{x z}^{i j} E_{z}^{j}, \\
& E_{z}^{i}=E_{f z}^{i}+M_{z x}^{i j} E_{x}^{j}+M_{z z}^{i j} E_{z}^{j},
\end{aligned}
$$

where we have adopted the convention that repeated superscripts in the same term are to be summed over. In Eqs. (2.14), $\mathbf{E}^{i}$ and $\mathbf{E}_{f}^{i}$ are the total and incident fields at the center $\left(x_{i}, z_{i}\right)$ of the $i$ th cell, the cells $i$ and $j$ are restricted to the region $\Omega_{2}$ occupied by the particle, and the matrix elements $M_{\mu \nu}^{i j}$ are given by

$$
M_{\mu \nu}^{i j}=k_{0}^{2}\left[\epsilon\left(x_{j}, z_{j}\right)-\epsilon_{f}\left(z_{j}\right)\right] G_{\mu v}^{i j}
$$

with

$$
G_{\mu v}^{i j}=\int_{x_{j}-(1 / 2) \Delta x}^{x_{j}+(1 / 2) \Delta x} d x^{\prime} \int_{z_{j}-(1 / 2) \Delta z}^{z_{j}+(1 / 2) \Delta z} d z^{\prime} G_{\mu v}\left(x_{i}-x^{\prime} ; z_{i}, z^{\prime}\right)
$$

In the numerical calculations carried out in this paper we have set $\Delta z$ equal to $\Delta x$.

The calculation of $G_{\mu \nu}^{i j}$ is far from straightforward, due to the singularity of $\overleftrightarrow{G}\left(x-x^{\prime} ; z, z^{\prime}\right)$ when $(x, z)=\left(x^{\prime}, z^{\prime}\right)$, and the pole its Fourier coefficient with respect to $x-x^{\prime}$ possesses at the wave number $k_{\text {sp }}(\omega)$ of the surface plasmon polariton of frequency $\omega$. This calculation is described in detail in Ref. 17.

The solution of Eqs. (2.14) gives the components of the total electric field in the region $\Omega_{2}$ occupied by the surface defect. Once that is known, the field at any point outside this region can be obtained from Eqs. (2.14) by allowing the point $\left(x_{i}, z_{i}\right)$ to be any point outside this region, while the sum over $j$ still runs over the cells within this region. 


\section{Calculation of the near field and the far field}

\section{The near field}

As we have already noted, once the system of equations (2.14) has been solved, the electric field inside the particle is known. It is then straightfoward to calculate the near field, i.e., the total electric field within a few wavelengths $\lambda$ of the surface, where $\lambda$ is defined in terms of the frequency of the surface plasmon polariton by $\lambda=2 \pi c / \omega$. Indeed, for this purpose we use the same set of equations (2.14), but now assume the point $\left(x_{i}, z_{i}\right)$ to be outside the region of the surface defect, in the near field region, while the sum on $j$ runs over the cells within the region of the defect. It should be noted that the calculation of the near field does not require the inversion of any matrices.

\section{The far field}

In calculating the far field we require the scattered field far from the surface defect. We do not determine this field at some set of points $\left\{x_{i}, z_{i}\right\}$, but instead calculate the differential reflection coefficient (DRC). This is defined in terms of the power scattered into the angular interval $(\theta, \theta+d \theta)$ about the scattering angle $\theta, P_{\text {sc }}(\theta) d \theta$, and the power in the incident surface plasmon polariton $P_{\text {inc }}$ by

$$
\frac{\partial R}{\partial \theta}=\frac{P_{\mathrm{sc}}(\theta)}{P_{\mathrm{inc}}} .
$$

The total incident flux crossing the plane $x=$ const. per unit time per unit width of the system in the $y$ direction is

$$
P_{\text {inc }}=\int_{-\infty}^{\infty} d z \operatorname{Re}_{x}^{c},
$$

where $\mathbf{S}^{c}$ is the complex Poynting vector,

$$
\mathbf{S}^{c}=\frac{c}{8 \pi} \mathbf{E} \times \mathbf{H}^{*} \text {. }
$$

When we use the results given by Eqs. (2.10), (2.11), and (2.13), we find that

$$
P_{\mathrm{inc}}=\frac{\omega k_{\mathrm{sp}}}{16 \pi}\left(E_{\mathrm{sp}}^{(0)}\right)^{2}\left(\frac{\epsilon_{1}}{\beta_{1}^{3}}+\frac{\epsilon_{3}}{\beta_{3}^{3}}\right) .
$$

To calculate $\boldsymbol{P}_{\mathrm{sc}}(\theta)$ we start with the expression for the scattered field obtained from Eq. (2.8),

$$
\begin{array}{rl}
\mathbf{E}_{s}(x, z \mid \omega)=k_{0}^{2} \int_{\Omega_{2}} & d x^{\prime} d z^{\prime} \overleftrightarrow{G}\left(x-x^{\prime} ; z, z^{\prime}\right) \\
& \times\left[\epsilon\left(x^{\prime}, z^{\prime}\right)-\epsilon_{f}\left(z^{\prime}\right)\right] \mathbf{E}\left(x^{\prime}, z^{\prime} \mid \omega\right) .
\end{array}
$$

We next introduce the Fourier representation of the Green function $\overleftrightarrow{G}\left(x-x^{\prime} ; z, z^{\prime}\right)$

$$
\overleftrightarrow{G}\left(x-x^{\prime} ; z, z^{\prime}\right)=\frac{i}{4 \pi} \int_{-\infty}^{\infty} d k \overleftrightarrow{g}\left(k \mid z, z^{\prime}\right) \exp \left[i k\left(x-x^{\prime}\right)\right]
$$

An explicit result for the dyad $\overleftrightarrow{g}\left(k \mid z, z^{\prime}\right)$ is derived in Ref. 17.

Since we have assumed that the region $\Omega_{2}$ is always in the half-space $z>0$, the integration variable $z^{\prime}$ in Eq. (2.20) is always non-negative, $z^{\prime} \geq 0$. When $z$ is larger than $z^{\prime}$, or when $z$ is negative, $\overleftrightarrow{g}\left(k \mid z, z^{\prime}\right)$ has the forms

$$
\begin{aligned}
\overleftrightarrow{\boldsymbol{g}}\left(k \mid z, z^{\prime}\right) & =\overleftrightarrow{\boldsymbol{g}}^{>}\left(k \mid z^{\prime}\right) \exp \left[i \gamma_{1}(\omega) z\right], \quad z>z^{\prime} \\
& =\overleftrightarrow{\boldsymbol{g}}^{<}\left(k \mid z^{\prime}\right) \exp \left[-i \gamma_{3}(\omega) z\right], \quad z<0,
\end{aligned}
$$

where $(i=1,3)$

$$
\begin{aligned}
\gamma_{i}(\omega) & =\left[\epsilon_{i} k_{0}^{2}-k^{2}\right]^{1 / 2}, \quad k^{2}<\epsilon_{i} k_{0}^{2} \\
& =i\left[k^{2}-\epsilon_{i} k_{0}^{2}\right]^{1 / 2}, \quad k^{2}>\epsilon_{i} k_{0}^{2} .
\end{aligned}
$$

The use of Eqs. (2.21) and (2.22) in Eq. (2.20), and a change in the order of integration yields the following representation of the scattered field:

$$
\begin{aligned}
\mathbf{E}_{s}(x, z \mid \omega) & =\int_{-\infty}^{\infty} d k \mathbf{e}_{s}^{>}(k) \exp \left(i k x+i \gamma_{1} z\right), \quad z>z_{2} \\
& =\int_{-\infty}^{\infty} d k \mathbf{e}_{s}^{<}(k) \exp \left(i k x-i \gamma_{3} z\right), \quad z<0
\end{aligned}
$$

where $z_{2}$ is the largest ordinate in $\Omega_{2}$, and

$$
\begin{aligned}
\overleftrightarrow{\mathrm{e}}_{s}^{\gtrless}(k)=\frac{i k_{0}^{2}}{4 \pi} \int_{\Omega_{2}} d x^{\prime} d z^{\prime} \overleftrightarrow{\mathrm{g}} \gtrless\left(k \mid z^{\prime}\right) e^{-i k x^{\prime}} \\
\quad \times\left[\epsilon\left(x^{\prime}, z^{\prime}\right)-\epsilon_{f}\left(z^{\prime}\right)\right] \mathbf{E}\left(x^{\prime}, z^{\prime} \mid \omega\right) .
\end{aligned}
$$

With the use of the Maxwell equation $\nabla \times \mathbf{E}=-c^{-1} \partial \mathbf{H} / \partial t$, the magnetic component of the scattered field can be written in the form

$$
\begin{aligned}
\mathbf{H}_{s}(x, z \mid \omega) & =\int_{-\infty}^{\infty} d k \mathbf{h}_{s}^{>}(k) \exp \left(i k x+i \gamma_{1} z\right), \quad z>z_{2} \\
& =\int_{-\infty}^{\infty} d k \mathbf{h}_{s}^{<}(k) \exp \left(i k x-i \gamma_{3} z\right), \quad z<0,
\end{aligned}
$$

where

$$
\begin{aligned}
& \mathbf{h}_{s}^{>}(k)=\hat{y} \frac{c}{\omega}\left[\gamma_{1} e_{s x}^{>}(k)-k e_{s z}^{>}(k)\right]=\frac{\omega}{c \gamma_{1}} \epsilon_{1} e_{s x}^{>}, \\
& \mathbf{h}_{s}^{<}(k)=\hat{y} \frac{c}{\omega}\left[-\gamma_{3} e_{s x}^{<}(k)-k e_{s z}^{<}(k)\right]=-\frac{\omega}{c \gamma_{3}} \epsilon_{3} e_{s x}^{<},
\end{aligned}
$$

since $\nabla \cdot \mathbf{E}=0$ in the far field region.

We can now proceed to the calculation of $P_{\mathrm{sc}}(\theta)$. We will consider explicitly only the case in which the region $\Omega_{1}(z>0)$ is a lossless metal, and the region $\Omega_{3}(z<0)$ is a lossless dielectric. The case in which $\Omega_{1}$ is a lossless dielectric and $\Omega_{3}$ is a lossless metal is treated in exactly the same way.

In the case being considered, the scattered field, which has the form of a bulk wave, is nonzero only in the region $\Omega_{3}$. The time average of the $z$ component of the Poynting vector of the scattered field in the region $\Omega_{3}(z<0)$ is 


$$
\begin{aligned}
\left\langle S_{z}\right\rangle & =\operatorname{Re} S_{z}^{c}=\frac{c}{8 \pi} \operatorname{Re} E_{s x} H_{s y}^{*} \\
& =\operatorname{Re}\left[-\frac{i c^{2}}{8 \pi \omega \epsilon_{3}} \frac{\partial H_{s y}}{\partial z} H_{s y}^{*}\right] .
\end{aligned}
$$

The total scattered flux crossing a plane $z=$ const. $<0$ per unit time per unit width of the system in the $y$ direction is

$$
\begin{aligned}
P_{\mathrm{sc}} & =\int_{-\infty}^{\infty} d x\left\langle S_{z}\right\rangle \\
& =-\frac{c^{2}}{4 \omega \epsilon_{3}} \int_{-\sqrt{\epsilon_{3}} k_{0}}^{\sqrt{\epsilon_{3} k_{0}}} d k \gamma_{3}\left|h_{s y}^{<}(k)\right|^{2} .
\end{aligned}
$$

We now introduce the scattering angle $\theta$, measured counterclockwise from the $-z$ axis, by

$$
k=\sqrt{\epsilon_{3}} k_{0} \sin \theta .
$$

It follows that $\gamma_{3}=\sqrt{\epsilon_{3}} k_{0} \cos \theta$ and $d k=\sqrt{\epsilon_{3}} k_{0} \cos \theta d \theta$. The magnitude of $P_{\text {sc }}$ can then be rewritten as

$$
\left|P_{\mathrm{sc}}\right|=\int_{-\pi / 2}^{\pi / 2} d \theta P_{\mathrm{sc}}(\theta)
$$

where

$$
P_{s c}(\theta)=\frac{\omega}{4} \cos ^{2} \theta\left|h_{s y}^{<}\left(\sqrt{\epsilon_{3}} k_{0} \sin \theta\right)\right|^{2} .
$$

The differential reflection coefficient is, therefore, given by

$$
\begin{aligned}
\frac{\partial R_{p}}{\partial \theta} & =4 \pi \frac{\cos ^{2} \theta\left|h_{s y}^{<}\left(\sqrt{\epsilon_{3}} k_{0} \sin \theta\right)\right|^{2}}{k_{\mathrm{sp}}\left[\frac{\epsilon_{1}}{\beta_{1}^{3}}+\frac{\epsilon_{3}}{\beta_{3}^{3}}\right]\left(E_{\mathrm{sp}}^{(0)}\right)^{2}} \\
& =\frac{4 \pi \epsilon_{3}\left|e_{s x}^{<}\left(\sqrt{\epsilon_{3}} k_{0} \sin \theta\right)\right|^{2}}{k_{\mathrm{sp}}\left[\frac{\epsilon_{1}}{\beta_{1}^{3}}+\frac{\epsilon_{3}}{\beta_{3}^{3}}\right]\left(E_{\mathrm{sp}}^{(0)}\right)^{2}}
\end{aligned}
$$

The amplitudes $e_{s x}^{<}(k)$ and $e_{s z}^{<}(k)$ can be calculated from Eq. (2.25) once the electric field in the source region is known, and $h_{s y}^{<}(k)$ is obtained from them by the use of Eq. (2.27b).

\section{The surface plasmon polariton reflection, transmission, and scattering coefficients}

When the incident surface plasmon polariton impinges on the particle, three kinds of waves are generated: (i) a transmitted surface plasmon polariton; (ii) a reflected surface plasmon polariton, and (iii) bulk electromagnetic waves which propagate away from the region of the particle in the lossless dielectric medium. It is of interest to calculate the fraction $R$ of the power in the incident surface plasmon polariton that is converted into the reflected surface plasmon polariton; the fraction $T$ transmitted beyond the particle in the form of a surface plasmon polariton; and the fraction $S$ converted into bulk waves.

Knowledge of the differential reflection coefficient enables us to evaluate $S$. Indeed, from Eqs. (2.16) and (2.31) we obtain

$$
S=\frac{P_{\mathrm{sc}}}{P_{\mathrm{inc}}}=\frac{1}{P_{\mathrm{inc}}} \int_{-\pi / 2}^{\pi / 2} d \theta P_{\mathrm{sc}}(\theta)=\int_{-\pi / 2}^{\pi / 2} d \theta \frac{\partial R_{p}}{\partial \theta}
$$

To calculate the surface plasmon polariton reflection and transmission coefficients, $R$ and $T$, respectively, we first notice from Eqs. (2.10) and (2.11) that a surface plasmon polariton is completely defined once we know its sense of propagation and the value of $E_{x}(z \mid \omega)$ at one point. Moreover, the power flow in a surface plasmon polariton is proportional to $\left|E_{\mathrm{sp}}\right|^{2}$, where $E_{\mathrm{sp}}$ is the value of $E_{x}(x, z \mid \omega)$ at $(x, z)=(0,0)$. We also note that since the metals we consider in this paper are lossless, the modulus of any component of the electric field of a surface plasmon polariton is a function only of $z$. Thus we have

$$
R=\left|\frac{E_{\mathrm{sp}}^{(R)}}{E_{\mathrm{sp}}^{(0)}}\right|^{2}, \quad T=\left|\frac{E_{\mathrm{sp}}^{(T)}}{E_{\mathrm{sp}}^{(0)}}\right|^{2},
$$

where $E_{\mathrm{sp}}^{(R)}\left(E_{\mathrm{sp}}^{(T)}\right)$ is the amplitude of the $x$ component of the electric field of the reflected (transmitted) surface plasmon polariton at $z=0$.

The near field scattered by the particle has two contributions: (1) the contribution of the surface waves; (2) the contribution of the bulk waves, which vanishes rapidly with increasing $z$ inside the metal. For the calculation of $R$ and $T$ it is only the former contribution that is of interest. Once the electric field inside the particle is known we can calculate the scattered field taking into account only this contribution. ${ }^{17}$ The complete derivation is given in Sec. III of Ref. 17. The resulting scattered field has the form of the field of a surface plasmon polariton on each side of the particle. We use this scattered field to calculate $R$ and $T$.

Thus, for the calculation of $R$ we calculate the incident field and the surface polariton contribution to the scattered field $\mathbf{E}_{s}(x, z \mid \omega)$ at the same point $\left(x_{i}, z_{i}\right)$ in the metal in front of the particle, and obtain

$$
R=\left|\frac{E_{\mathrm{sp}}^{(R)}}{E_{\mathrm{sp}}^{(0)}}\right|^{2}=\left|\frac{E_{s x}\left(x_{i}, z_{i} \mid \omega\right)}{E_{f x}\left(x_{i}, z_{i} \mid \omega\right)}\right|^{2}
$$

In the same way we calculate the incident field and the total field at the same point $\left(x_{i}, z_{i}\right)$ in the metal behind the particle, and obtain

$$
T=\left|\frac{E_{\mathrm{sp}}^{(T)}}{E_{\mathrm{sp}}^{(0)}}\right|^{2}=\left|\frac{E_{x}\left(x_{i}, z_{i} \mid \omega\right)}{E_{f x}\left(x_{i}, z_{i} \mid \omega\right)}\right|^{2} .
$$

\section{RESULTS}

In this article, we focus our attention on the case of long particles, i.e., particles whose length is much larger than their width. This is, for example, the case for a film of finite length deposited on a substrate or embedded in the substrate. For such a long particle we expect to see some features in the results of our scattering calculations that can be related to the properties of surface and guided wave polaritons propagating in the corresponding threeor four-layer system that is obtained when the length of 
the particle becomes infinite (cf. Fig. 2). From the dispersion relations of these waves (Appendixes A and B) we find that at a given frequency these multilayer systems support zero, one, or two surface plasmon polaritons, and zero, one, or more guided wave polaritons. Thus, when the incident surface plasmon polariton impinges on the particle, it excites one or more of these surface and guided waves. Then, since the waves that are excited are reflected from the end of the particle, this system acts as a Fabry-Perot interferometer. When the length $L$ of the particle is increased the surface polariton reflection coefficient $R$ should display an oscillatory dependence on $L$. This has been discussed by Leskova and Gapotchenko, ${ }^{14}$ but in our paper we are not restricted to very long particles. Finally, we can look at the near field above the particle. Here, too, we should see some interference patterns related to the geometry of the problem and the difference in dielectric constants. In view of current activity in near field optics, such a result might be of interest as a way obtaining information about the dielectric constant of finite films.

In this article, we will restrict our analysis to three general cases. In Sec. III A we will study the scattering of a surface plasmon polariton from long dielectric particles deposited on a metal. We will first obtain the dispersion curves of surface guided waves in the equivalent threelayer system, which will aid in explaining the results for scattering from a particle of finite length. In Sec. III B we will focus on a metallic inclusion in a metal. In Sec. III C we will discuss the case of a dielectric particle embedded in a metal.
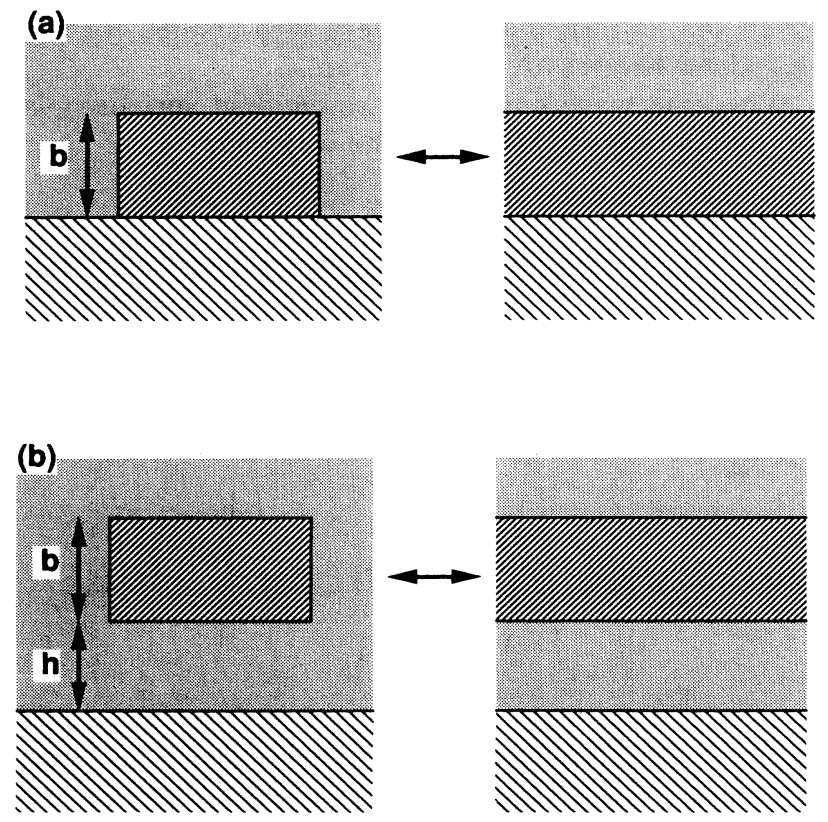

FIG. 2. (a) A film of finite length on a metallic substrate and the corresponding infinite, three-layer system, (b) a particle embedded in a metallic substrate and the corresponding infinite four-layer system.

\section{A. Dielectric particle on a metallic substrate}

\section{Dispersion curves for the corresponding three-layer system}

We consider a slab of thickness $d$ between two semiinfinite media. The dielectric constant of this system, $\epsilon(z)$, is given by

$$
\epsilon(z)= \begin{cases}\epsilon_{1} & \text { for } z>d \\ \epsilon_{2} & \text { for } 0<z<d \\ \epsilon_{3} & \text { for } z<0 .\end{cases}
$$

We derive in Appendix A the dispersion relation for surface and guided wave polaritons propagating in this system. It is a transcendental equation that must be solved numerically. In this subsection we assume that the upper semi-infinite medium is vacuum $\left(\epsilon_{1}=1\right)$; that the slab is a lossless dielectric $\left(\epsilon_{2}=\epsilon_{d}\right)$; and that the lower semiinfinite medium is a lossless metal whose dielectric constant has the Drude form $\left[\epsilon_{3}=1-\left(\omega_{p}^{2} / \omega^{2}\right)\right.$, where $\omega_{p}$ is the plasma frequency of the charge carriers].

We present in Fig. 3 a typical example of the resulting dispersion curves. We can observe the dispersion curve of the surface polariton propagating along the interface between the slab and the metal. This curve tends to an asymptotic value of $\left(\omega / \omega_{p}\right)=1 / \sqrt{\epsilon_{d}+1}$ [i.e., $\epsilon(\omega)=-\epsilon_{d}$ ]. In addition, we can see the dispersion curves of guided wave polaritons propagating in the slab. We note that for $\left(\omega / \omega_{p}\right)<1 / \sqrt{\epsilon_{d}+1}$ a polariton can propagate along this three-layer system, but for $1 / \sqrt{\epsilon_{d}+1}<\left(\omega / \omega_{p}\right)<1 / \sqrt{2}$ no polariton can propagate along this system, although a polariton can propagate along a single metal-vacuum interface. The presence of additional guided waves is linked to the ratio of the

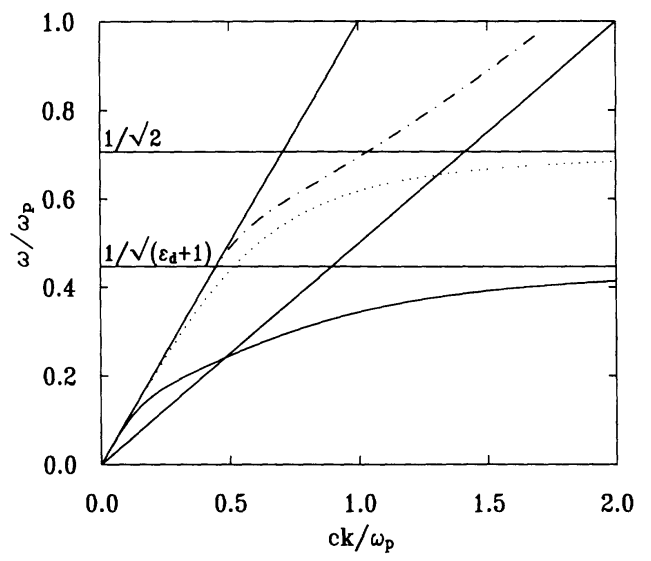

FIG. 3. Dispersion curves for a three-layer system consisting of vacuum for $z>d$, a dielectric slab of dielectric constant $\epsilon_{d}$ for $0<z<d$, and a lossless metal, whose dielectric function has the Drude form, for $z<0$. For this graph $d / \lambda_{p}=0.5$ (with $\left.\omega_{p} / c=2 \pi / \lambda_{p}\right)$, and $\epsilon_{d}=4$. Solid curve: surface polariton dispersion curve; dotted line: surface polariton dispersion curve for a metal-vacuum interface; dot-dash curve: guided wave dispersion curve. 
height $d$ of the layer to the wavelength in vacuum, $\lambda=2 \pi c / \omega$.

\section{Dielectric particle of finite length on a metal substrate}

The preceding analysis of a three-layer system leads us to the following comments concerning the interaction of a surface plasmon polariton with a long dielectric particle on a metallic substrate. We first analyze the frequency range in which a surface plasmon polariton can propagate in the three-layer system, $\left(\omega / \omega_{p}\right)<1 / \sqrt{\epsilon_{d}+1}$. For frequencies in this range we expect that the incoming surface polariton will excite another surface polariton in the particle, whose wave number $k_{\mathrm{sp}}^{(2)}$ must be almost equal to that of a surface polariton in the corresponding threelayer system at the same frequency. This second polariton will be reflected from the right edge of the particle and, as a consequence, the near field above the particle should display an interference pattern with a spatial period $D$ that satisfies $2 k_{\mathrm{sp}}^{(2)}=2 \pi / D$.

Figure 4 displays the square of the electric field at an ordinate just above the dielectric particle. This dielectric particle has a dielectric constant $\epsilon_{d}=2.25$, while the dielectric constant of the substrate is -17.2 . The length of the particle $a$ is equal to $3 \lambda$, and its width $b$ is $\lambda / 10$. This particle is placed on the substrate between $x / \lambda=5$ and $x / \lambda=8$. On the left side of the particle we see an interference pattern due to the interference of the incident polariton with the reflected polariton due to the finite length of the particle. The period $D_{0}$ of this interference satisfies $2 k_{\mathrm{sp}}=2 \pi / D_{0} \cdot{ }^{17}$ By measuring this period $D_{0}$ we find a value of $k_{\mathrm{sp}}=(1.025 \pm 0.015)(\omega / c)$, which is to be compared with $k_{\mathrm{sp}}=1.03(\omega / c)$ given by Eq. (2.12a). In the same way the interference pattern above the particle is due to the interference of the second polariton propagating in the positive $x$ direction with the second polari-

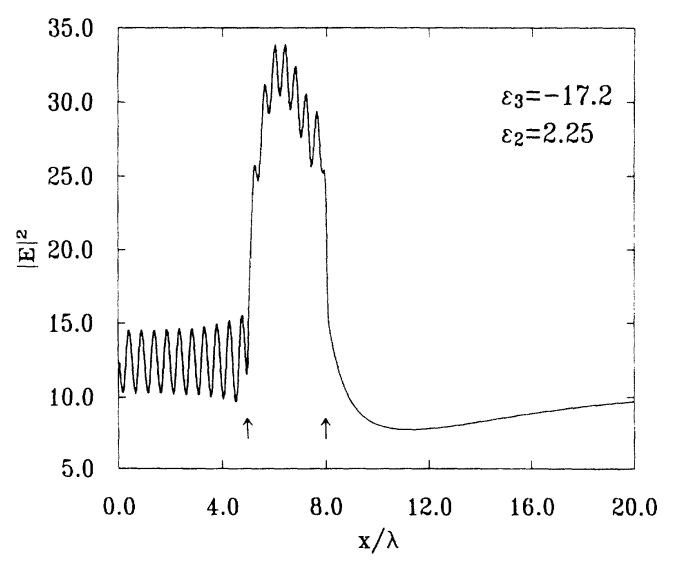

FIG. 4. Near field for a system consisting of a finite dielectric particle of length $3 \lambda$ and thickness $\lambda / 10$ deposited on a metallic substrate, where $\lambda$ is the wavelength in vacuum $(\lambda=2 \pi c / \omega)$. The dielectric constants are -17.2 for the metal and 2.25 for the dielectric. The near field displayed is the square of the modulus of the electric field at a fixed ordinate $z / \lambda=5 / 40$. The size of the integration mesh used is $\Delta x=\lambda / 20$. ton reflected from the right edge of the particle. From the period of this interference structure we obtain a value of $k_{\mathrm{sp}}^{(2)}$ equal to $(1.26 \pm 0.04)(\omega / c)$, which is to be compared with the theoretical value of $1.257(\omega / c)$ calculated numerically for the three-layer system. The agreement between these two values is very good, which indicates that a particle of length $3 \lambda$ supports the same surface polariton as the infinite three-layer system. Thus it is possible to evaluate $k_{\mathrm{sp}}^{(2)}$ by looking at the near field. From a knowledge of $k_{\mathrm{sp}}^{(2)}$ and the height of the defect it should be possible to estimate $\epsilon_{2}$, the dielectric constant of the dielectric cylinder, by the use of the results of Appendix A. As a consequence, looking at the near field should be of interest in determining dielectric constants of integrated optics elements, since it is not obvious how to evaluate, by conventional methods, the dielectric constant of a dielectric cylinder whose cross-section dimensions are of the order of a micron.

A particle on a substrate possesses some similarities with a slab in a Fabry-Perot interferometer. Thus we expect to see an oscillatory dependence of the reflection coefficient $R$ on the length $a$ of the particle. Such an interference pattern has already been found by Leskova and Gapotchenko $^{14}$ for the case of a very long particle $\left(\left[k_{\mathrm{sp}}^{(2)}-(\omega / c)\right] a>1\right)$. In this paper we find an interference pattern with a period $\Delta a_{R}=2 \pi / 2 k_{\mathrm{sp}}^{(2)}$ for much smaller values of the length $a$. Figure 5 displays the $R$ coefficient for the system used in obtaining Fig. 3 but as a function of the length $a$. The conservation of energy is very well satisfied at each point since $|1-R-S-T|<10^{-4}$ (and most of the time $<10^{-6}$ ). We see a very well-defined interference structure for the reflection coefficient, which yields a value of $k_{\mathrm{sp}}^{(2)}$ equal to $(1.253 \pm 0.02)(\omega / c)$, in good agreement with the theoretical value of $1.257(\omega / c)$. It should be noted that the interference pattern is already very well-defined for particles whose lengths are smaller than $\lambda$.

When the wave number of the incident surface polariton differs only slightly from the wave number of light in

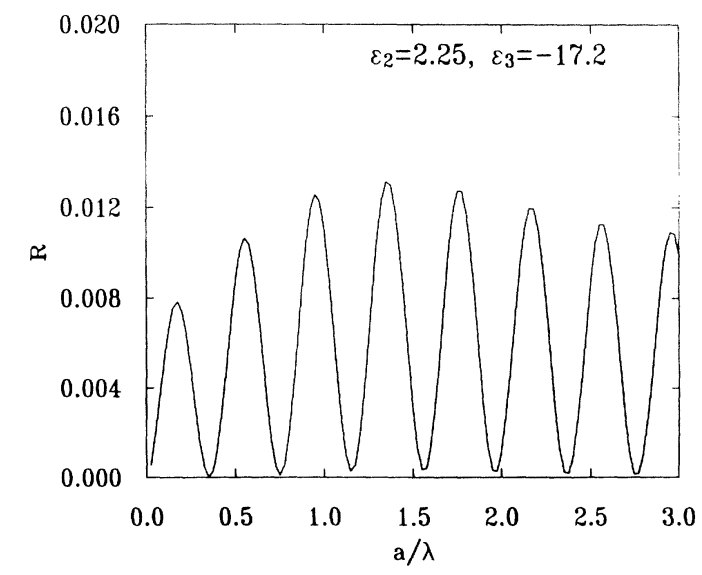

FIG. 5. Reflection coefficient for a system consisting of a dielectric particle on a metallic substrate as a function of the length of the particle $a$. The parameters are: thickness $b=\lambda / 10, h=0$ (the particle touches the substrate), $\epsilon_{2}=2.25$, $\epsilon_{3}=-17.2$, and $\Delta x=\lambda / 40$. 
vacuum, the field scattered into bulk waves at the first edge of the particle has a maximum power flow into directions almost parallel to the surface. This can be seen in Fig. 6, which shows the DRC for a particle of length $3 \lambda$ and height $\lambda / 20$ of dielectric constant 2.25 on a metallic surface. We display the DRC for the dielectric constant of the metal $\epsilon_{m}=-5$ (dotted line) and -17.2 (solid line). For $\epsilon_{m}=-17.2, k_{\mathrm{sp}}$ is equal to $1.03(\omega / c)$, also close to $(\omega / c)$, and the maximum of the scattered energy is in the forward direction. For comparison, when $\epsilon_{m}=-5, k_{\mathrm{sp}}$ is equal to $1.12(\omega / c)$, and the maximum of the scattered energy is no longer in the direction parallel to the surface.

These bulk waves can excite a surface polariton at the right edge of the particle. As a consequence, when $\left[\left(k_{\mathrm{sp}}-(\omega / c)\right] a \ll 1\right.$ we have two sources for the transmitted polariton: the bulk waves and the second polariton propagating along the particle-metal interface. Thus we expect an oscillatory dependence of the transmission coefficient $T$ on the length $a$ of the particle. The period $\Delta a_{T}$ of this interference pattern should satisfy $2 \pi / \Delta a_{T}=k_{\mathrm{sp}}^{(2)}-(\omega / c)$. This behavior has already been predicted by Leskova and Gapotchenko ${ }^{14}$ for the case of a metallic film on a metallic substrate.

Figure 7 displays the reflection coefficient $R$ and transmission coefficient $T$ as functions of the length $a$ of a film whose dielectric constant is 4 and whose height is $\lambda / 10$, deposited on a metallic substrate of dielectric constant -8 . As in Fig. 5, we can see an interference pattern for the reflection coefficient. The measured period of these interferences yields a value of $(2.53 \pm 0.1)(\omega / c)$ for the wave number $k_{\mathrm{sp}}^{(2)}$ of the surface polariton, in good agreement with the theoretical value of $2.545(\omega / c)$. Moreover, we see an interference pattern for the transmission coefficient as expected. The measured value of the period, although a little less precise, yields a value of $(2.57 \pm 0.1)(\omega / c)$ for $k_{\mathrm{sp}}^{(2)}$, in agreement with the previ-

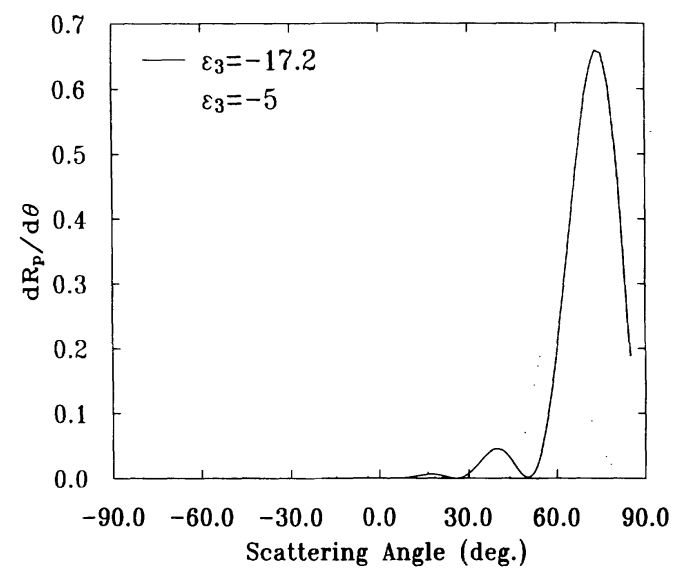

FIG. 6. Differential reflection coefficient for a system consisting of a dielectric particle over a metallic substrate for two values of the substrate dielectric constant. The parameters are $a=3 \lambda, b=\lambda / 20, \epsilon_{2}=2.25$, and $\Delta x=\lambda / 40$.

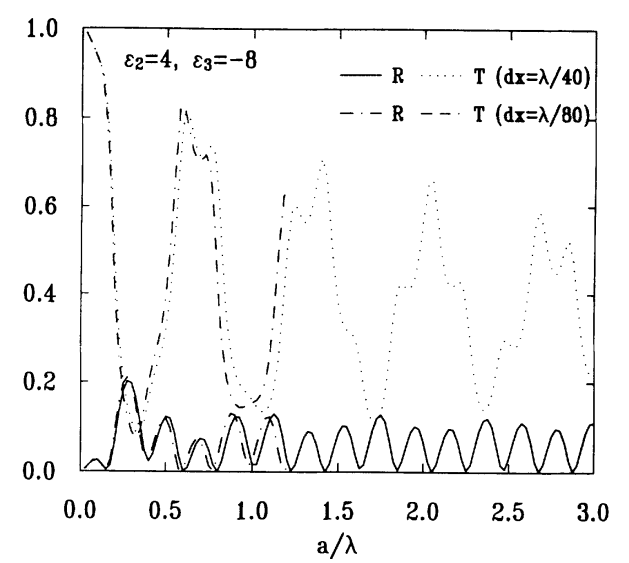

FIG. 7. Reflection and transmission coefficients for a system consisting of a dielectric particle on a metallic substrate. $b=\lambda / 10, \epsilon_{2}=4$, and $\epsilon_{3}=-8$. Two sizes of the integration mesh have been used: $\Delta x=\lambda / 40$ and $\Delta x=\lambda / 80$.

ous values. We note that these results have been obtained for particles whose lengths are smaller than $3 \lambda$. We also note that when $a$ increases the amplitude of the oscillations of the transmission coefficient diminishes, since the power flow of bulk waves reaching the second edge of the particle is smaller the larger $a$ is.

In Fig. 7, we have displayed the reflection coefficient $R$ and transmission coefficient $T$ for two values of the mesh size $\Delta x$. For the solid and dotted curves $\Delta x$ is equal to $\lambda / 40$, whereas for the dash-dot and dash-dash-dot curves $\Delta x$ is equal to $\lambda / 80$. Although these curves are almost identical, we can see that the period of the interference is slightly shorter when $\Delta x$ is equal to $\lambda / 80$. This is due to the fact that $k_{\mathrm{sp}}^{(2)}$ is large, thus a finer discretization of the field within the particle is required. We note that the values of $k_{\mathrm{sp}}^{(2)}$ have been calculated with the results obtained for $\Delta x$ equal to $\lambda / 80$. In contrast, although the result for $R$ presented in Fig. 5 was calculated with $\Delta x=\lambda / 40$, no difference in this result was observed when the calculations were repeated with $\Delta x=\lambda / 80$, because of the much smaller value of $k_{\mathrm{sp}}^{(2)}$ in this case.

In the case that $1 / \sqrt{\epsilon_{d}+1}<\omega / \omega_{p}<1 / \sqrt{2}$, no surface polariton can propagate along the three-layer system. As a consequence, when the incident surface polariton impinges on the particle it cannot excite any surface polariton. The main source for the transmitted polariton is then the bulk waves due to the diffraction of the incident surface polariton from the left edge of the particle. Consequently, we can expect a sharp decrease of the transmission coefficient with increasing length of the particle due to the decrease of the bulk wave power flow reaching the right edge of the particle. Another possible source for the transmitted polariton is the existence of guided waves when the height of the particle is large enough. However, we are not able to display in this article results for this range of frequencies, since we could not obtain converged results, i.e., when the mesh size $\Delta x$ was decreased, the results for the reflection, transmission, and scattering coefficients changed significantly. 


\section{B. Metallic inclusion in a metallic host}

\section{Dispersion curves for the corresponding three-layer system}

In this subsection we are concerned with a long metallic particle located in a different metal and interfacing with a semi-infinite vacuum. As we have seen in the preceding subsection, it is worthwhile studying the corresponding three-layer system defined by

$$
\epsilon(z)=\left\{\begin{array}{l}
\epsilon(\omega) \text { for } z>d \\
\epsilon_{2}(\omega) \text { for } 0<z<d \\
1 \text { for } z<0 .
\end{array}\right.
$$

The metallic dielectric functions are assumed to have the Drude form, viz., $\epsilon(\omega)=1-\left(\omega_{p}^{2} / \omega^{2}\right), \epsilon_{2}(\omega)=1$ $-\left(\omega_{p 2}^{2} / \omega^{2}\right)$, and we denote by $\alpha$ the ratio $\omega_{p 2} / \omega_{p}$. Note that the vacuum now occupies the region $z<0$, which makes the corresponding experiment less easy to carry out. We were obliged to make this choice since our computational approach is based on the assumption that the defect is situated in the upper semi-infinite medium. However, we can easily imagine that the $z$ direction points downward to obtain a more conventional picture of the system.

We display in Fig. 8 the dispersion curves for the structure defined by Eq. (3.2) for two values of $\alpha$, viz. $\alpha=0.4$ [Fig. 8(a)] and $\alpha=1.5$ [Fig. 8(b)]. For values of $\alpha$ smaller than 1 [Fig. 8(a)] two dispersion curves can be seen. The lower one is the dispersion curve of the surface polariton at the slab-vacuum interface. The asymptotic value of $\omega / \omega_{p}$ reached by this curve is $\alpha / \sqrt{2}$. This value corresponds to a frequency $\omega_{p 2} / \sqrt{2}$, which is the usual asymptotic value for the case of a planar interface between two semi-infinite media: vacuum and a metal whose dielectric function is $\epsilon_{2}(\omega)$. In this frequency range $k_{\mathrm{sp}}^{(2)}$ has very large values. As a consequence, the skin depth for the polariton is very small and the polariton does not feel the presence of the semi-infinite metallic medium. This explains why we retrieve the results for a planar interface between two semi-infinite media. The upper dispersion curve corresponds to a surface polariton propagating along the interface between the slab and the semi-infinite metallic medium. Indeed, when $\alpha$ is smaller than one, there is a range of frequencies in which the dielectric function of the slab is positive, while the dielectric function of the semi-infinite metallic medium remains negative. Thus a polariton can propagate along the interface between these two media.

For values of $\alpha$ larger than 1 [Fig. 8(b)] we have similar results, but now the asymptotic value of $\omega / \omega_{p}$ for the lower branch is larger than $1 / \sqrt{2}$, and for the upper curve the dielectric function of the slab is negative whereas the dielectric function of the semi-infinite metallic medium is positive.

\section{Long metallic inclusion in a metallic substrate}

The preceding analysis of the dispersion curves prompts the following comments. When the plasma fre- quency of the metal in the slab is larger than that of the semi-infinite metal substrate $(\alpha>1)$, then the situation is simple for the entire range of frequencies in which a surface polariton can propagate along the planar metalvacuum interface $\left(\omega / \omega_{p}<1 / \sqrt{2}\right)$. Indeed, in this case there is always one, and only one, surface polariton that can propagate along the three-layer system. For $\alpha<1$ the situation is more complicated since there are ranges of frequencies in which either one or two surface polaritons can propagate along the three-layer system when $\omega / \omega_{p}$ is smaller than $1 / \sqrt{2}$. We look at a case where only one polariton can propagate, and follow in that case the analysis of the previous subsection. We display in Fig. 9 the reflection and scattering coefficients for a metallic inclusion of dielectric constant -5 in a metallic host of dielectric constant -2 . The metallic inclusion is in contact with the vacuum, its thickness is $\lambda / 20$, and its length $a$ varies from 0 to $3 \lambda$. Since the dielectric constant in the inclusion is smaller than that of the metallic host, this case corresponds to a value of $\alpha$ larger than one.
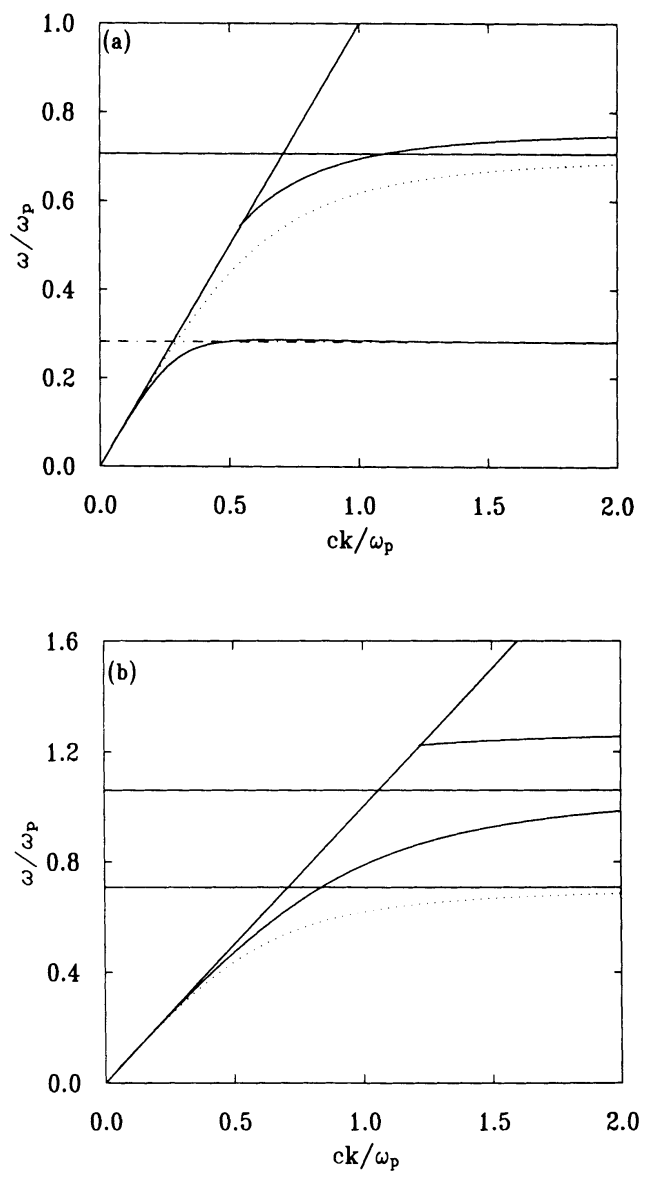

FIG. 8. Dispersion curves for a three-layer system consisting of vacuum for $z<0$, a metallic slab whose dielectric function is $\epsilon_{2}=1-\omega_{p 2}^{2} / \omega^{2}$ for $0<z<d$, and a metal whose dielectric function is $\epsilon_{1}=1-\omega_{p}^{2} / \omega^{2}$ for $z>d$ (solid curve). The dotted curve represents the dispersion curve for a surface polariton in the structure without the slab. (a) $d=\lambda_{p} / 5, \omega_{p 2} / \omega_{p}=0.4$ and (b) $d=\lambda_{p}, \omega_{p 2} / \omega_{p}=1.5$. 


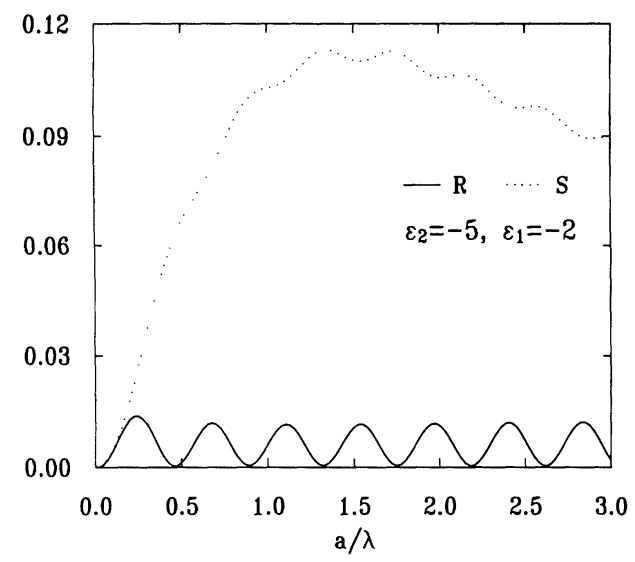

FIG. 9. Reflection and scattering coefficients for a system consisting of a metallic inclusion $\left(\epsilon_{2}=-5\right)$ in a metal host $\left(\epsilon_{1}=-2\right)$, when the inclusion touches the interface $(h=0)$. The other parameters are $b=\lambda / 20$ and $\Delta x=\lambda / 40$.

One polariton can propagate along the corresponding three-layer system. As a consequence, a similar surface polariton is excited at the left edge of the inclusion and is reflected from the right edge. Thus we observe an interference pattern for the reflection coefficient as a function of $a$, with the same origin as that for the result displayed in Fig. 5. The value of $k_{\mathrm{sp}}^{(2)}$ given by our scattering calculation is $(1.153 \pm 0.008)(\omega / c)$, which is in very good agreement with the value of $k_{\mathrm{sp}}^{(2)}$ for the threelayer system, viz., $1.143(\omega / c)$. Once again we find these interferences even for very small value of $a$.

For the same reason as in Sec. III A 2, we should also be able to see an interference pattern for the transmission coefficient as the length of the sample increases. The period of the interference $\Delta a_{T}$ is $2 \pi /\left[k_{\mathrm{sp}}^{(2)}-(\omega / c)\right]$, so that in order to obtain a small period we must choose a frequency yielding a large value of $k_{\mathrm{sp}}^{(2)}$, that is a value of $\omega / \omega_{p}$ slightly smaller than $\alpha / \sqrt{2}$. We display in Fig. 10 the results for such a frequency. The dielectric constant of the inclusion (the host) is $-1.2(-3.4)$. The reflection coefficient as a function of $a$ shows an interference pattern, with a short period $\Delta a_{R}$, which yields a large value

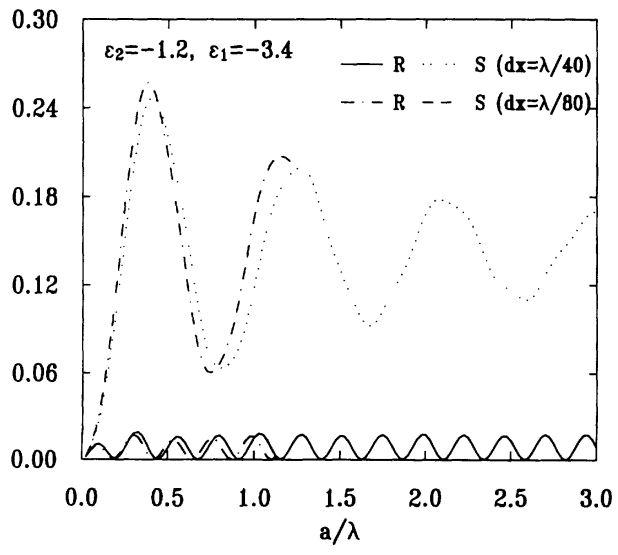

FIG. 10. Same as Fig. 9 with $\epsilon_{1}=-3.4, \epsilon_{2}=-1.2$, and $b=\lambda / 10$. Two sizes for the integration mesh have been used $\Delta x=\lambda / 40$ and $\Delta x=\lambda / 80$. of $k_{\mathrm{sp}}^{(2)}=(2.2 \pm 0.08)(\omega / c)$, in good agreement with the theoretical value for the three-layer system of $k_{\mathrm{sp}}^{(2)}=2.22(\omega / c)$. We display the scattering coefficient instead of the transmission coefficient in order to plot the two curves $(R$ and $S)$ on the same graph. Since $R+T+S=1$, and $R$ takes very small values, we have $S \simeq 1-T$, and the interference pattern for $S$ is the same as the one for $T$. The period of these interferences yields a value of $(2.27 \pm 0.08)(\omega / c)$ for $k_{\mathrm{sp}}^{(2)}$.

As in Fig. 7, we have displayed in Fig. 10 the results for two sizes of the elementary mesh $\Delta x$. We find discrepancies similar to those observed in Fig. 7. Indeed, since $k_{\mathrm{sp}}^{(2)}$ is large, we need a finer mesh to solve the equations accurately. The choice of $\Delta x=\lambda / 40$ enables us to deal with large systems with a reasonable amount of computer time, and the results are of interest since they differ only slightly from those of a more accurate solution.

Finally, we display in Fig. 11 the near field for this configuration when the length of the inclusion is equal to $2 \lambda$. The results are not as clear as those presented in Fig. 4. Indeed, over the defect (in vacuum) we have an interference between three waves: the bulk wave and two surface polaritons, one propagating in the positive $x$ direction, the other in the negative $x$ direction. Since the prominent wave consists of the surface polariton propagating in the positive $x$ direction, we expect to see the interference of this polariton with the polariton propagating in the negative $x$ direction with a period $\Delta_{\mathrm{sp}}=\pi / k_{\mathrm{sp}}^{(2)}$, and the interference of this polariton with the bulk wave with a period $\Delta_{\text {bulk }}=2 \pi /\left[k_{\mathrm{sp}}^{(2)}-(\omega / c)\right]$. In Fig. $4, \Delta_{\text {bulk }}$ was larger than the size of the particle, and this second type of interference could not be observed. The situation is different for the results plotted in Fig. 11 where $\Delta_{\text {bulk }} \simeq 0.808 \lambda$ and $\Delta_{\text {sp }}=0.2235 \lambda$. Although the analysis of the results displayed in this figure becomes somewhat tedious, we were able to find a value of $(0.226 \pm 0.007) \lambda$ for $\Delta_{\mathrm{sp}}$, which yields a value for $k_{\mathrm{sp}}^{(2)}$ of $(2.21 \pm 0.061)(\omega / c)$.

The interferences with the bulk wave are not very clear in Fig. 11. Nevertheless, by looking carefully at this

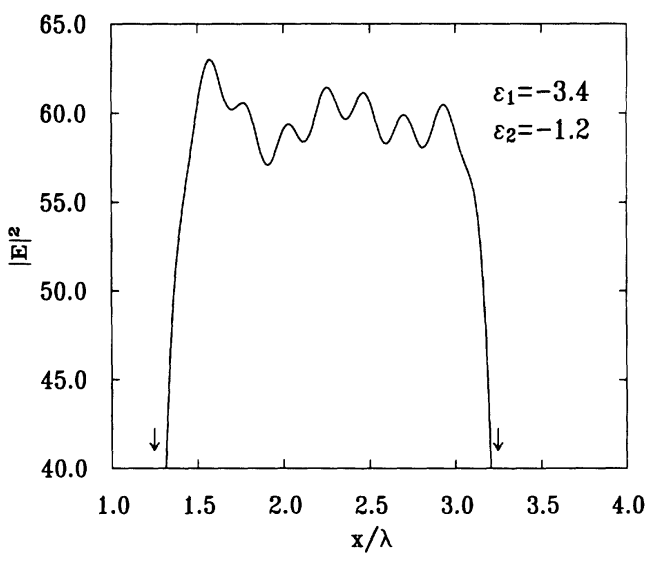

FIG. 11. Near field for the same system used in obtaining Figs. 10 and 11. $a=2 \lambda, b=\lambda / 40, \Delta x=\lambda / 80, \epsilon_{1}=-3.4$, and $\epsilon_{2}=-1.2$. The near field displayed is the square of the modulus of the electric field at a fixed ordinate $z=-\lambda / 160$. The arrows indicate the edges of the particle. 
figure we can notice oscillations with a large period, with the first main dip around $x / \lambda=1.9$ and a second dip around $x / \lambda=2.7$. This second dip does not appear clearly due to the fact that at the same position we have a peak due to the interference between the two surface polaritons. From the distance between these two dips we find $\Delta_{\text {bulk }}=(0.797 \pm 0.06) \lambda$, which yields a value for $k_{\mathrm{sp}}^{(2)}$ of $(2.25 \pm 0.07)(\omega / c)$.

Since we cannot describe fully every possible case, we refer to the article of Leskova and Gapotchenko ${ }^{14}$ for the study of the frequency range where two polaritons can propagate along the three-layer system. For frequencies in the gap, i.e., in the frequency range in which no surface polariton can propagate along the three-layer system, we encountered problems of convergence similar to those described in the preceding subsection.

\section{Dielectric inclusion within a metallic host}

\section{Dispersion curves for the corresponding four-layer system}

As we have seen in Secs. III A and III B, knowledge of the dispersion curves of surface and guided wave polaritons in the corresponding multilayer structure is of primary importance when we want to study the scattering of a surface plasmon polariton by a long particle. In contrast to the systems studied in the two preceding subsections, in this subsection we study the scattering of surface plasmon polaritons by particles completely embedded in the host. As a consequence, the extrapolated system for an infinitely long particle is a four-layer system [Fig. 2(b)]. We derive in Appendix B the dispersion relation for such a structure. It is obvious that, even by restricting ourselves to structures consisting of dielectric and metallic layers, a completely general study of this dispersion relation is outside the scope of this article. Consequently, we will limit ourselves to the case of a dielectric layer in a metal host.

For that case we consider a system whose dielectric constant $\epsilon(z)$ satisfies

$$
\epsilon(z)= \begin{cases}\epsilon_{1} & \text { for } z>d_{2} \\ \epsilon_{2} & \text { for } d_{1}<z<d_{2} \\ \epsilon_{1} & \text { for } 0<z<d_{1} \\ \epsilon_{3} & \text { for } z<0,\end{cases}
$$

where $\epsilon_{2}$ and $\epsilon_{3}$ are two positive real dielectric constants, and $\epsilon_{1}$ has the Drude form $\epsilon_{1}=1-\left(\omega_{p}^{2} / \omega^{2}\right)$. We display in Fig. 12 the dispersion curves for a typical set of parameters: $\epsilon_{3}=1, \epsilon_{2}=16, d_{1}=0.105 \lambda_{p}$, and $d_{2}=0.211 \lambda_{p}$. As expected, we observe three branches. The two lowerfrequency branches correspond to the surface polaritons propagating along the interfaces between the dielectric layer and the metal. These two branches converge towards a value of $\omega / \omega_{p}=1 / \sqrt{\epsilon_{2}+1}$. The third (highestfrequency) branch corresponds to the surface polariton propagating along the vacuum-metal interface.

In this example no guided wave polariton dispersion curve is present in Fig. 12 due to the small width of the dielectric layer.

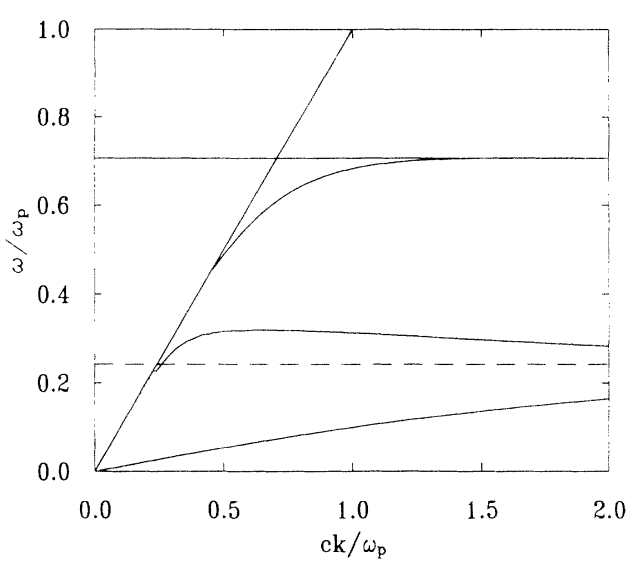

FIG. 12. Dispersion curves for a four-layer system consisting of vacuum for $z<0$, a metal whose dielectric function is $\epsilon=1-\omega_{p}^{2} / \omega^{2}$ for $0<z<d_{1}$ and $z>d_{2}$, and a dielectric slab for $d_{1}<z<d_{2}$. We have $d_{1}=0.105 \lambda_{p}, d_{2}=0.211 \lambda_{p}$, and $\epsilon_{d}=16$. The solid curve represents the different surface polariton dispersion curves. The dotted line is the surface polariton dispersion curve for a similar system without the dielectric slab.

In the absence of guided waves, we can distinguish three frequency domains. For low frequencies two surface polaritons can propagate. For frequencies just below $\omega_{p} / \sqrt{2}$ only one surface polariton can propagate. And for the range of frequencies in between a gap appears in which no surface polariton can propagate.

\section{Dielectric inclusion of finite length within a metallic host}

We turn now to the problem of the scattering of a surface plasmon polariton by a long dielectric particle embedded in the metal. We will consider here the cases where one or no surface polariton can propagate in the corresponding four-layer system. We omit consideration of the case in which two surface polaritons can propagate in the four-layer system, since the study of the near field and the reflection coefficient in this case would be too long for this article.

We turn first to the case where only one surface polariton can propagate in the corresponding four-layer system. We note that this case corresponds to a value of the frequency close to $\omega_{p} / \sqrt{2}$, which means a rather small modulus for the dielectric constant, at least smaller than the value of the dielectric constant of the dielectric inclusion. In a similar manner as in the two preceding subsections, where only one surface polariton could propagate in the three-layer system considered there, we expect to see an interference pattern for the surface polariton reflection coefficient as a function of the length of the inclusion. We have displayed in Fig. 13 this reflection coefficient for a system characterized by $\epsilon_{1}=-3.472$, $\epsilon_{2}=16, \epsilon_{3}=1, b=\lambda / 20$ ( $b$ is the width of the particle), and $h=\lambda / 20$ ( $h$ is its distance into the metal).

We can see as expected the interference pattern for $R$. The period of these interferences yields a value of $k_{\text {sp }}=(1.018 \pm 0.006)(\omega / c)$, which is in very good agreement with the theoretical value of $k_{\mathrm{sp}}^{(2)}=1.017(\omega / c)$. We 


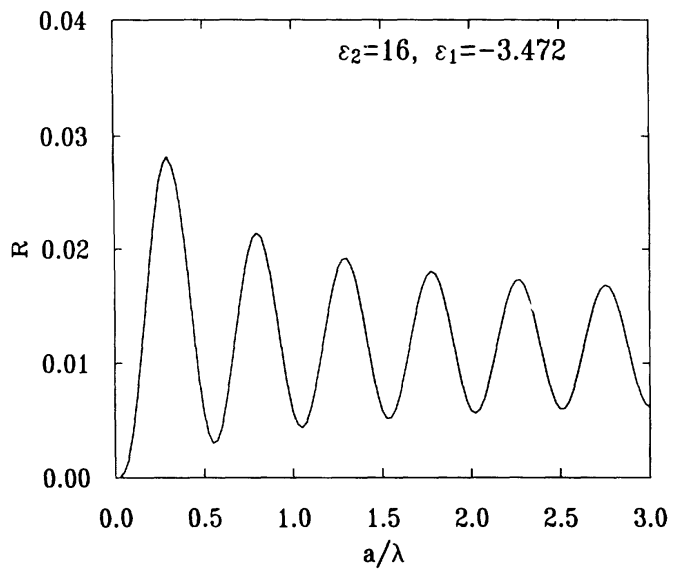

FIG. 13. Reflection coefficient for a system consisting of a dielectric particle totally embedded in a metal. $\epsilon_{1}=-3.472$, $\epsilon_{2}=16, b=\lambda / 20, h=\lambda / 20$, and $\Delta x=\lambda / 40$.

note that since the value of the modulus of $\epsilon(\omega)$ is small, $k_{\text {sp }}$ differs significantly from the wave number of light in vacuum, and as a consequence (cf. Fig. 6) the bulk waves excited at the first edge of the particle have a maximum power flow direction significantly away from the forward horizontal direction. This means that it will be hard to see an interference pattern for the transmission coefficient as the length of the particle is increased unless we find parameters yielding a large value for $k_{\mathrm{sp}}^{(2)}$.

By looking at the near field along the $x$ axis we expect to see an interference pattern as in Fig. 4 and for the same physical reason. Figure 14 displays the near field along the $x$ axis in vacuum but very close to the metal. The edges of the particle are marked by arrows. We observe the interference pattern and, by analyzing it, we find a value of $k_{\mathrm{sp}}^{(2)}=(1.01 \pm 0.02)(\omega / c)$, correct but less precise than the value found by looking at the interference pattern for $R$. Moreover, the intensity of the electric field on the vertical sides of the particle is larger than over the particle, and the amplitude of the interferences

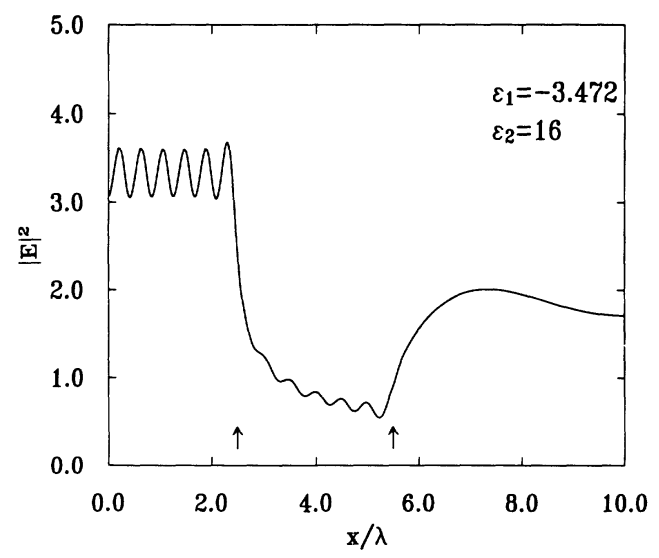

FIG. 14. Near field for the same system used in obtaining Fig. 13. $a=3 \lambda$. The near field displayed is the square of the modulus of the electric field at a constant value of $z: z=-\lambda / 80$. above the particle is not very large. Consequently, it will not be easy to characterize this sample by looking at the near field: only the edges of the inclusion could be detected easily.

When no polariton can propagate along the four-layer system, we expect no interference pattern in the reflection coefficient or the near field for finite-size particles. Figure 15 displays the reflection and transmission coefficients as functions of the length $a$ of the particle. The parameters for this plot are $\epsilon_{1}=-3.472, \epsilon_{2}=10, \epsilon_{3}=1$ and $h=b=\lambda / 20$. The reflection coefficient tends to a constant value of $8 \%$ as the length of the particle increases, whereas the transmission coefficient decreases sharply. This sharp decrease can be interpreted as follows. The main source for the transmitted surface polariton, especially for a long particle, is usually the polariton propagating along the finite three- or four-layer system whose wave number has been denoted by $k_{\mathrm{sp}}^{(2)}$ throughout this paper. But in the present case there is no such second surface polariton, and the only remaining source for the transmitted polariton consists of the bulk waves excited at the first edge of the defect, which excite the transmitted polariton at the second edge of the particle. Since the power flow of the bulk waves reaching the second edge diminishes when the length of the particle increases, the transmission coefficient follows the same trend.

If we turn to a study of the near field, we expect, as already said, no interference pattern for the electric field above the particle. Figure 16 displays the squared modulus of the electric field when the parameters are the same as for Fig. 15, the length of the particle is two vacuum wavelengths $(a=2 \lambda)$, and we calculate $|E(x, z \mid \omega)|^{2}$ in the vacuum just above the interface $(z=-\lambda / 160)$. The arrows on the graph show the edges of the particle. We observe the usual interference pattern in front of the particle due to the interference between the incoming and reflected surface polaritons, but no interference above the particle, since no surface polaritons can propagate back and forth. We cannot obtain a precise value of the dielectric constant of the inclusion by looking at the near field, but we can infer the positions of the ends of the particle and can obtain some idea of the dielectric constant, since we know we are in the gap of the dispersion curves.

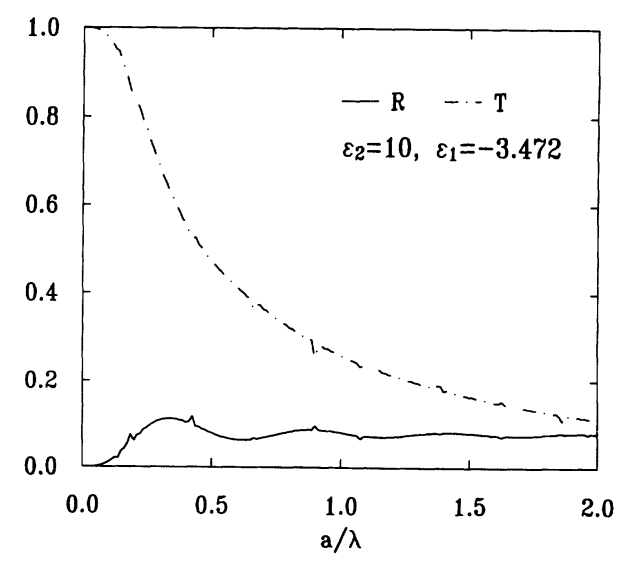

FIG. 15. Reflection and transmission coefficients for the system used in obtaining Fig. 13, with $\epsilon_{2}=10$ and $\Delta x=\lambda / 80$. 


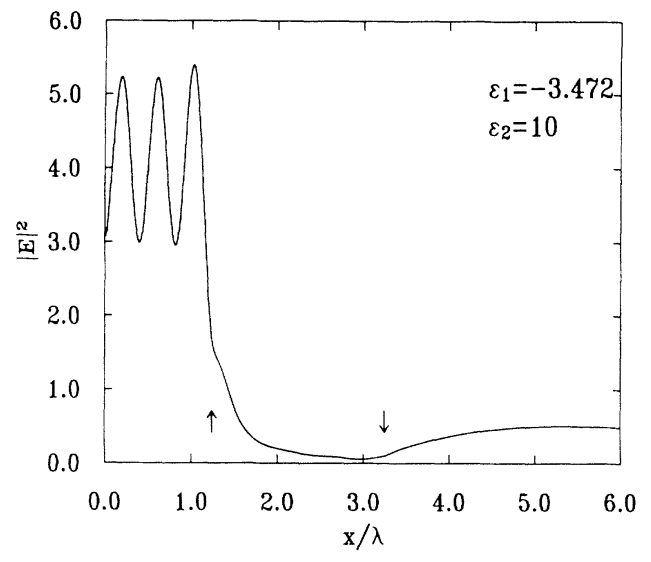

FIG. 16. Same as Fig. 14 with $a=2 \lambda, \Delta x=\lambda / 80, \epsilon_{2}=10$, and $z=-\lambda / 160$.

\section{CONCLUSIONS}

We have developed a numerical approach to the scattering of a $p$-polarized surface electromagnetic wave by a surface defect that is based on a volume integral equation formulation of the scattering problem. This approach enables us to compute the transmission and reflection coefficients for the surface wave as well as the far field and near field. We have focused our attention on the cases of defects whose length is much larger than their width. It has been shown that the knowledge of the dispersion curves for the corresponding multilayer system (i.e., the system in which the defect has an infinite length) plays a key role in the understanding of the physical phenomena arising in the scattering process. This is already true for defects whose lengths are only of the order of $\lambda=2 \pi c / \omega$, where $\omega$ is the frequency of the surface polariton. We have seen that zero, one, or two surface plasmon polaritons, together with guided electromagnetic waves, can propagate along the defect. In looking at the reflection and transmission coefficients as functions of the length of the particle, one sees an interference structure that yields information about the wave vectors of the surface waves, and thus about the dielectric constant of the defect. The same information can be inferred by looking at the near field, which is of more practical interest. This might have promising applications in integrated optics as, for example, a way of finding the edges of submerged waveguides or of probing the dielectric constant of a waveguide whose geometry is known.

\section{ACKNOWLEDGMENTS}

The work of A.A.M. was supported in part by National Science Foundation Grant No. DMR-9319404. F.P. would like to thank the Saint-Gobain Company for support. This research was also supported by the University of California, Irvine, through an allocation of computer time.

\section{APPENDIX A: DISPERSION RELATION FOR $p$-POLARIZED SURFACE POLARITONS IN A THREE-LAYER SYSTEM}

We consider a three-layer system whose dielectric constant is defined by

$$
\epsilon(z)= \begin{cases}\epsilon_{1} & \text { for } z>d \\ \epsilon_{2} & \text { for } 0<z<d \\ \epsilon_{3} & \text { for } z<0\end{cases}
$$

In $p$ polarization the magnetic field has only a single, nonzero component, $\mathbf{H}(\mathbf{x} \mid \omega)=\left[0, H_{y}(x, z \mid \omega), 0\right]$, when the plane of incidence is the $x z$ plane, and the amplitude $H_{y}(x, z \mid \omega)$ for a surface wave propagating in the positive $x$ direction can be written as follows:

$$
H_{y}(x, z \mid \omega)=\left\{\begin{array}{l}
A e^{i k x-\beta_{1} z}, \quad z>d \\
e^{i k x}\left[B e^{\beta_{2} z}+C e^{-\beta_{2} z}\right], \quad 0<z<d \\
D e^{i k x+\beta_{3} z}, \quad z<0,
\end{array}\right.
$$

with $(i=1,2,3)$

$$
\beta_{i}=\left(k^{2}-\epsilon_{i} \frac{\omega^{2}}{c^{2}}\right)^{1 / 2}, \quad \operatorname{Re} \beta_{i}>0, \quad \operatorname{Im} \beta_{i} \leq 0 .
$$

The boundary conditions satisfied by $H_{y}(x, z \mid \omega)$ require the continuity of $H_{y}$ and $\epsilon^{-1} \partial H_{y} / \partial z$ across each interface. These conditions at $z=d$ yield the pair of equations

$$
\begin{aligned}
A e^{-\beta_{1} d} & =B e^{\beta_{2} d}+C e^{-\beta_{2} d}-\frac{\beta_{1}}{\epsilon_{1}} A e^{-\beta_{1} d} \\
& =\frac{1}{\epsilon_{2}}\left(B e^{\beta_{2} d}-C e^{-\beta_{2} d}\right)
\end{aligned}
$$

and at $z=0$ we obtain

$$
\begin{aligned}
& D=B+C, \\
& \frac{1}{\epsilon_{3}} \beta_{3} D=\frac{1}{\epsilon_{2}} \beta_{2}(B-C) .
\end{aligned}
$$

The solvability condition for the system of Eqs. (A4) - (A5) yields the dispersion relation for surface polaritons in the structure defined by Eq. (A1)

$$
\begin{aligned}
\frac{1}{\epsilon_{3}} \beta_{3} & {\left[\left(1-\frac{\epsilon_{2} \beta_{1}}{\epsilon_{1} \beta_{2}}\right] e^{-\beta_{2} d}+\left(1+\frac{\epsilon_{2} \beta_{1}}{\epsilon_{1} \beta_{2}}\right) e^{\beta_{2} d}\right] } \\
& -\frac{1}{\epsilon_{2}} \beta_{2}\left[\left(1-\frac{\epsilon_{2} \beta_{1}}{\epsilon_{1} \beta_{2}}\right) e^{-\beta_{2} d}-\left(1+\frac{\epsilon_{2} \beta_{1}}{\epsilon_{1} \beta_{2}}\right) e^{\beta_{2} d}\right]=0 .
\end{aligned}
$$

\section{APPENDIX B: DISPERSION RELATION FOR $p$-POLARIZED SURFACE POLARITONS IN A FOUR-LAYER SYSTEM}

We consider a four-layer system whose dielectric constant is given by 


$$
\epsilon(z)= \begin{cases}\epsilon_{1}, & z>d_{2} \\ \epsilon_{2}, & d_{1}<z<d_{2} \\ \epsilon_{3}, & 0<z<d_{1} \\ \epsilon_{4}, & z<0 .\end{cases}
$$

The single, nonzero component of the magnetic field for a surface polariton propagating in the positive $x$ direction in this structure is given by

$$
H_{y}(x, z \mid \omega)=\left\{\begin{array}{l}
A e^{i k x-\beta_{1} z}, \quad z>d_{2} \\
e^{i k x}\left(B e^{\beta_{2} z}+C e^{-\beta_{2} z}\right), d_{1}<z<d_{2} \\
e^{i k x}\left(D e^{\beta_{3} z}+F e^{-\beta_{3} z}\right), 0<z<d_{1} \\
G e^{i k x+\beta_{4} z}, z<0,
\end{array}\right.
$$

where $(i=1,2,3,4)$

$$
\beta_{i}=\left(k^{2}-\epsilon_{i} \frac{\omega^{2}}{c^{2}}\right)^{1 / 2}, \quad \operatorname{Re} \beta_{i}>0, \quad \operatorname{Im} \beta_{i} \leq 0
$$

The boundary conditions require the continuity of $H_{y}$ and $\epsilon^{-1} \partial H_{y} / \partial z$ at each interface. To find the dispersion relation we proceed as in Appendix A. We write the equations arising from the satisfaction of the boundary conditions at $z=d_{2}, z=d_{1}$, and $z=0$. The solvability condition for the resulting system of equations yields the dispersion relation for surface polaritons in the structure defined by Eq. (B1)

$$
\begin{gathered}
\left(1-\frac{\epsilon_{2} \beta_{1}}{\epsilon_{1} \beta_{2}}\right) e^{-\beta_{2} d_{2}} e^{\beta_{2} d_{1}}\left[\left(1+\frac{\epsilon_{3} \beta_{2}}{\epsilon_{2} \beta_{3}}\right]\left[\frac{\beta_{4}}{\epsilon_{4}}-\frac{\beta_{3}}{\epsilon_{3}}\right) e^{-\beta_{3} d_{1}}+\left(1-\frac{\epsilon_{3} \beta_{2}}{\epsilon_{2} \beta_{3}}\right]\left[\frac{\beta_{4}}{\epsilon_{4}}+\frac{\beta_{3}}{\epsilon_{3}}\right] e^{\beta_{3} d_{1}}\right] \\
+\left(1+\frac{\epsilon_{2} \beta_{1}}{\epsilon_{1} \beta_{2}}\right] e^{\beta_{2} d_{2}} e^{-\beta_{2} d_{1}}\left[\left(1-\frac{\epsilon_{3} \beta_{2}}{\epsilon_{2} \beta_{3}}\right]\left[\frac{\beta_{4}}{\epsilon_{4}}-\frac{\beta_{3}}{\epsilon_{3}}\right] e^{-\beta_{3} d_{1}}+\left[1+\frac{\epsilon_{3} \beta_{2}}{\epsilon_{2} \beta_{3}}\right]\left[\frac{\beta_{4}}{\epsilon_{4}}+\frac{\beta_{3}}{\epsilon_{3}}\right] e^{\beta_{3} d_{1}}\right]=0 .
\end{gathered}
$$

*Permanent address: Laboratoire d'Enèrgétique Moléculaire et Macroscopique, Combustion, Ecole Centrale Paris, Châtenay-Malabry, 92295 Cedex, France.

${ }^{1}$ W. R. Dean, Proc. Cambridge Philos. Soc. 44, 483 (1948).

${ }^{2}$ F. Ursell, Proc. Cambridge Philos. Soc. 46, 141 (1950).

${ }^{3}$ F. Ursell, Proc. Cambridge Philos. Soc. 46, 153 (1950).

${ }^{4}$ H. Levine, J. Math. Phys. 6, 1231 (1965).

${ }^{5}$ A. M. J. Davis and M. J. Hood, SIAM J. Appl. Math. 31, 16 (1976).

${ }^{6}$ A. M. J. Davis and F. G. Leppington, Quart. J. Mech. Appl. Math. 32, 19 (1978).

${ }^{7}$ A. L. Cullen, Electron. Lett. 11, 479 (1975).

${ }^{8}$ A. M. J. Davis and F. G. Leppington, Proc. R. Soc. London Ser. A 353, 55 (1977).

${ }^{9}$ A. M. J. Davis and F. G. Leppington, Proc. R. Soc. London 358, 243 (1977).

${ }^{10}$ A. M. J. Davis, Proc. R. Soc. London Ser. A 391, 181 (1984).

${ }^{11}$ V. M. Agranovich, V. E. Kravtsov, and T. A. Leskova, Zh. Eksp. Teor. Fiz. 81, 1828 (1981) [Sov. Phys. JETP 54, 968 (1981)].

${ }^{12}$ V. M. Agranovich, V. E. Kravtsov, and T. A. Leskova, Solid State Commun. 40, 687 (1981).

${ }^{13}$ V. M. Agranovich, Zh. Eksp. Teor. Fiz. 77, 1124 (1979) [Sov. Phys. JETP 50, 567 (1979)].

${ }^{14}$ T. A. Leskova and N. I. Gapotchenko, Solid State Commun. 53, 351 (1985).

${ }^{15}$ G. I. Stegeman, A. A. Maradudin, T. P. Shen, and R. F. Wallis, Phys. Rev. B 29, 6530 (1984).
${ }^{16}$ T. P. Shen, R. F. Wallis, A. A. Maradudin, and G. I. Stegeman, Appl. Opt. 23, 607 (1984).

${ }^{17}$ F. Pincemin, A. Sentenac, and J.-J. Greffet, J. Opt. Soc. Am. A 11, 1117 (1994).

${ }^{18}$ F. Pincemin, A. Sentenac, and J.-J. Greffet (unpublished).

${ }^{19}$ Y. J. Chabel and A. J. Sievers, Appl. Phys. Lett. 32, 90 (1978).

${ }^{20} \mathrm{Z}$. Schlesinger and A. J. Sievers, Appl. Phys. Lett. 36, 409 (1980).

${ }^{21}$ Z. Schlesinger, B. C. Webb, and A. J. Sievers, Solid State Commun. 39, 1035 (1981).

${ }^{22}$ D. W. Pohl, in Scanning Tunneling Microscopy II, edited by R. Wiesenclanger and H.-J. Güntherodt (Springer-Verlag, Heidelberg, 1992), pp. 233-271.

${ }^{23}$ D. W. Pohl, in Near Field Optics, edited by D. W. Pohl and D. Courjon (Kluwer Academic, Dordrecht, 1993), pp. 1-5.

${ }^{24}$ D. Van Labeke and D. Barchiesi, in Near Field Optics, edited by D. W. Pohl and D. Courjon (Kluwer Academic, Dordrecht, 1993), p. 157-178.

${ }^{25}$ M. G. Cottam and D. R. Tilley, Introduction to Surface and Superlattice Excitations (Cambridge University Press, New York, 1989), p. 196.

${ }^{26}$ E. Burstein, A. Hartstein, J. Schoenwald, A. A. Maradudin, D. L. Mills, and R. F. Wallis, in Polaritons, edited by E. Burstein and F. de Martini (Pergamon, New York, 1974), pp. 89-108.

${ }^{27}$ R. F. Harrington, Field Computation by Moment Methods (Macmillan, New York, 1968). 


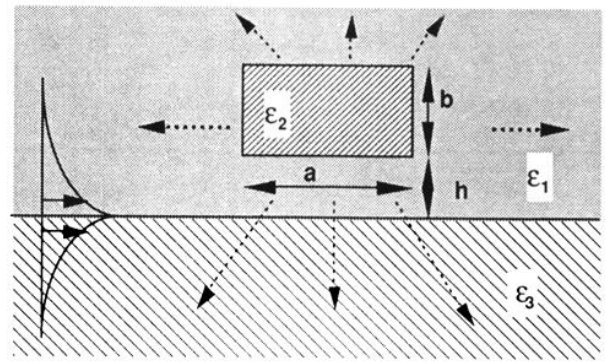

FIG. 1. Geometry of the system studied in this paper. 

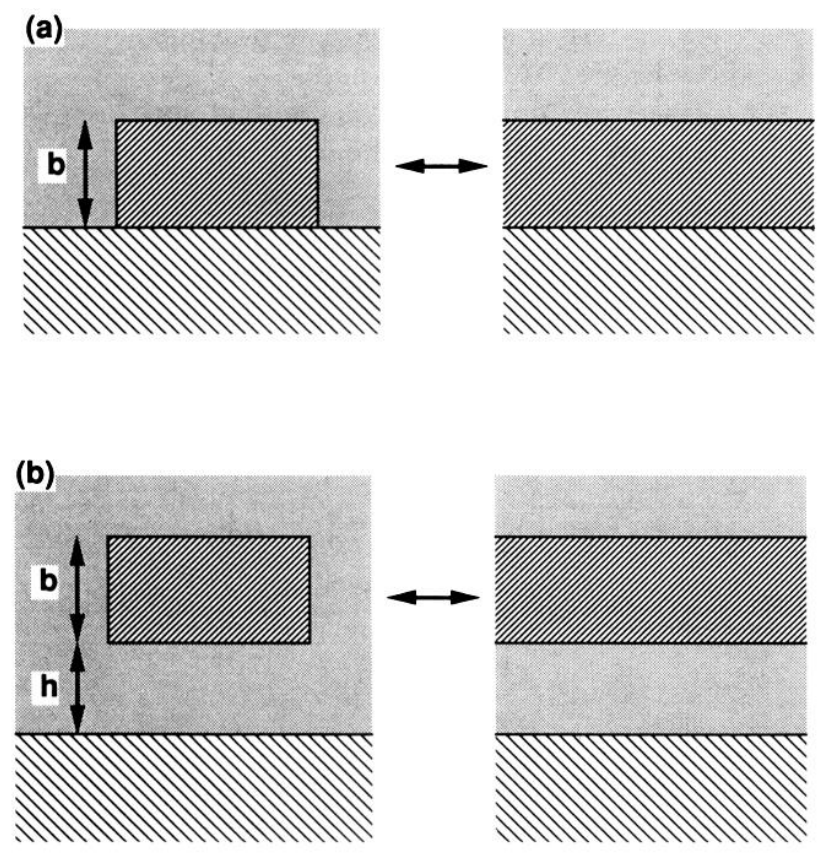

FIG. 2. (a) A film of finite length on a metallic substrate and the corresponding infinite, three-layer system, (b) a particle embedded in a metallic substrate and the corresponding infinite four-layer system. 